\title{
Chromatic Mechanisms in Striate Cortex of Macaque
}

\author{
Peter Lennie, John Krauskopf, ${ }^{a}$ and Gary Sclar \\ Center for Visual Science and Department of Psychology, University of Rochester, Rochester, New York 14627
}

\begin{abstract}
We measured the responses of $\mathbf{3 0 5}$ neurons in striate cortex to moving sinusoidal gratings modulated in chromaticity and luminance about a fixed white point. Stimuli were represented in a 3-dimensional color space defined by 2 chromatic axes and a third along which luminance varied. With rare exceptions the chromatic properties of cortical neurons were well described by a linear model in which the response of a cell is proportional to the sum (for complex cells, the rectified sum) of the signals from the 3 classes of cones. For each cell there is a vector passing through the white point along which modulation gives rise to a maximal response. The elevation $\left(\theta_{m}\right)$ and azimuth $\left(\phi_{m}\right)$ of this vector fully describe the chromatic properties of the cell. The linear model also describes neurons in l.g.n. (Derrington et al., 1984), so most neurons in striate cortex have the same chromatic selectivity as do neurons in I.g.n. However, the distributions of preferred vectors differed in cortex and I.g.n.: Most cortical neurons preferred modulation along vectors lying close to the achromatic axis and those showing overt chromatic opponency did not fall into the clearly defined chromatic groups seen in I.g.n. The neurons most responsive to chromatic modulation (found mainly in layers IVA, IVC $\beta$, and VI) had poor orientation selectivity, and responded to chromatic modulation of a spatially uniform field at least as well as they did to any grating. We encountered neurons with band-pass spatial selectivity for chromatically modulated stimuli in layers II/III and VI. Most had complex receptive fields. Neurons in layer II/III did not fall into distinct groups according to their chromatic sensitivities, and the chromatic properties of neurons known to lie within regions rich in cytochrome oxidase appeared no different from those of neurons in the interstices. Six neurons, all of which resembled simple cells, showed unusually sharp chromatic selectivity.
\end{abstract}

The principal aim of this paper is to provide a quantitative account of the chromatic properties of neurons in striate cortex of macaque and to show how these neurons transform the signals from the lateral geniculate nucleus (1.g.n.). Several earlier studies have examined the chromatic properties of neurons in striate cortex, either by measurement of their spectral sensitivities (Dow, 1974; Gouras, 1974; Michael, 1978a-c; Vautin and Dow, 1985) or by measurement of their responses to isoluminant chromatic

Received June 12, 1989; revised Sept. 25, 1989; accepted Sept. 28, 1989.

This work was supported by NIH grants EY 04440 to P.L., EY 01319 to the Center for Visual Science, and EY 06638 to J.K. J.K. was also supported by AFOSR-86-0334. G.S. held a National Research Service award.

Correspondence should be addressed to Peter Lennie at the above address.

aPresent address: Department of Psychology and Center for Neural Science New York University, New York, NY 10003.

Copyright (C) 1990 Society for Neuroscience $0270-6474 / 90 / 100649-21 \$ 02.00 / 0$ stimuli (Livingstone and Hubel, 1984; Thorell et al., 1984). Although responses to particular stimuli have been well characterized, this work has not given rise to a general account from which one can predict the responses of neurons to arbitrary chromatic stimuli, nor has it provided a quantitative account of how neurons combine signals from the 3 classes of cone. Derrington and colleagues (1984) showed that the response of a neuron in l.g.n. to any stimulus could be predicted from a simple model that assumed linear combination of signals from the 3 classes of cone. Here, we provide a similar analysis of the behavior of cortical neurons.

Our second purpose is to understand the relationship between the psychophysics of color vision and the properties of cortical neurons. Our earlier work on l.g.n. (Derrington et al., 1984) did not reveal 3 postreceptoral mechanisms of the kinds postulated on psychophysical grounds (an achromatic mechanism having high spatial acuity and 2 chromatically opponent ones of lower resolving power). Instead, we found 2 classes of parvocellular ncurons (P-cells), one in which signals from long-wavelengthsensitive $(R)$ cones were opposed to signals from the middlewavelength-sensitive $(G)$ cones and another in which signals from the short-wavelength-sensitive (B) cones were opposed to some combined signal from $R$ and $G$ cones. The $R-G$ neurons were overwhelmingly preponderant. Magnocellular neurons (M-cells) showed little chromatic opponency but, because they sample the image sparsely, are an improbable substrate of the putative achromatic mechanism. For virtually all P-cells the chromatically opponent mechanisms were segregated in center and surround of the receptive field. This attribute results in the chromatic properties of the neuron varying with the spatial properties of the stimulus (Wiesel and Hubel, 1966; DeValois et al., 1977; Ingling and Martinez-Uriegas, 1983), such that a preference for chromatically modulated stimuli at low spatial frequencies gives way to a preference for achromatic stimuli as spatial frequency is raised. Such neurons provide ambiguous information about the chromatic content of stimuli, although the ambiguity can be resolved by higher mechanisms that derive their inputs from the appropriate sets of neurons in 1.g.n. (D'Zmura and Lennie, 1986). In the present work we tried to establish whether such mechanisms exist in striate cortex.

\section{Materials and Methods}

Preparation and recording. These experiments were undertaken on 17 Macaca fascicularis that weighed between 2.5 and $4.5 \mathrm{~kg}$. Each animal was anesthetized initially with an injection of ketamine hydrochloride (Vetalar, $10 \mathrm{mg} / \mathrm{kg}$, i.m.) or (in some experiments) an injection of sufentanil citrate (Sufenta, 10-12 $\mu \mathrm{g} / \mathrm{kg}$, i.m.). Cannulae were inserted in the saphenous veins, and surgery was continued under sodium pentobarbital (Nembutal) or Sufenta anesthesia. In anesthetic doses Sufenta often produced severe respiratory depression, and animals required artificial respiration during surgery. Despite this, Sufenta appeared to be 

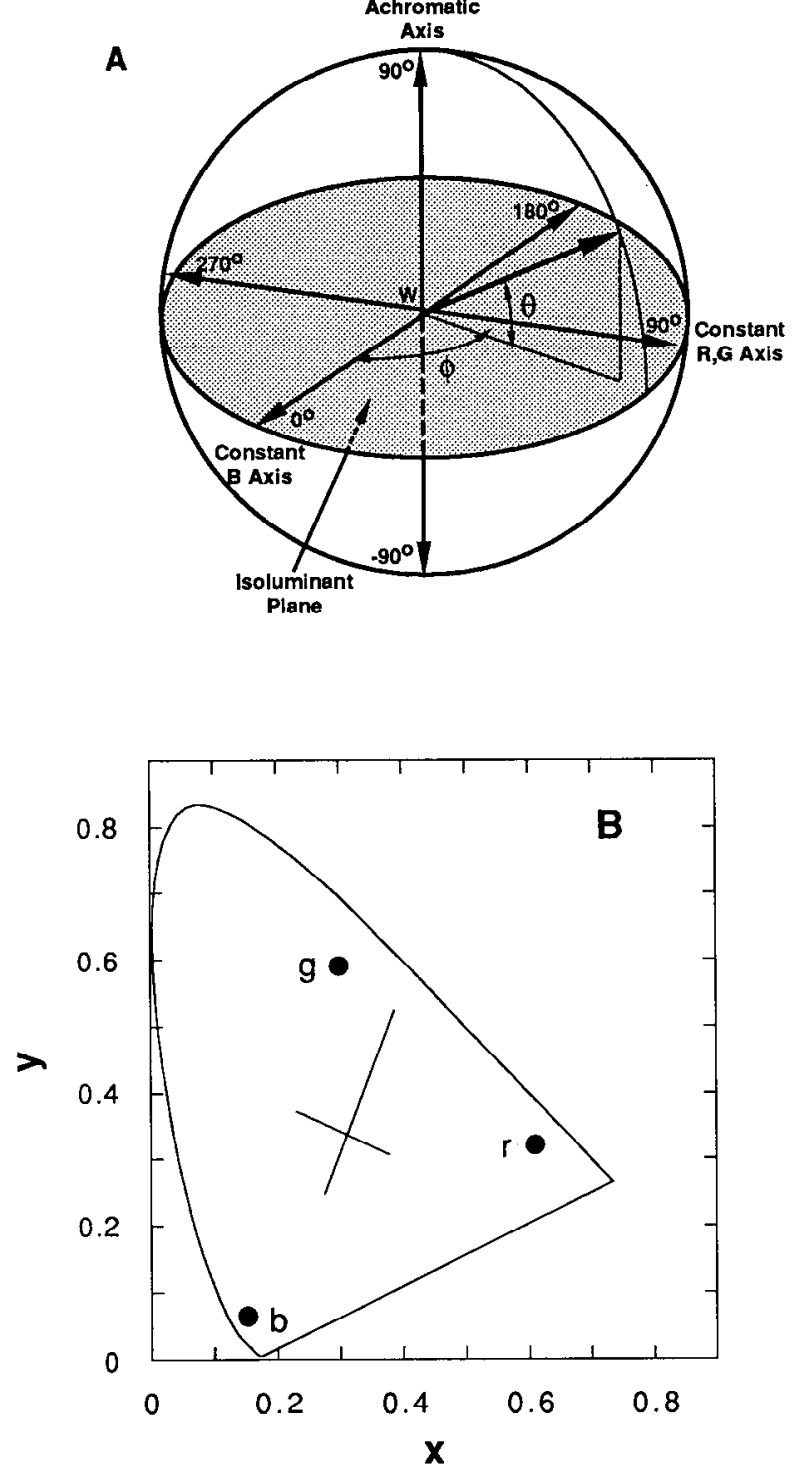

Figure 1. A, Color space in which stimuli were produced. An isoluminant plane is specified by the constant $B$ and constant $R, G$ axes, whose intersection ( $W$ ) defines the adaptation point (for the present experiments, white). The achromatic axis passes orthogonally through the isoluminant plane at the adaptation point. The direction of any modulation through this point is defined by its clevation $(\theta)$ with respect to the isoluminant plane and its azimuth $(\phi)$ with respect to the constant B axis. $\theta$ can take values between $-90^{\circ}$ and $90^{\circ} ; \phi$ can take values between $0^{\circ}$ and $360^{\circ} . B$, CIE chromaticity diagram showing the chromaticity coordinates of the television phosphors and the constant $B$ and constant $R, G$ axes. The adaptation point is defined by the intersection of the axes.

the more satisfactory anesthetic: In animals anesthetized with it neurons gave brisker responses and more were spontaneously active. It was used as an anesthetic in all later experiments.

The trachea was cannulated, the head placed in a stereotaxic frame and a craniotomy $8 \mathrm{~mm}$ in diameter was made above the representation of the fovea on striate cortex, centered about $5 \mathrm{~mm}$ behind the lunate sulcus. After the electrode carrier had been positioned, the hole was covered with warm agar and sealed with wax.

After surgery a loading dose of vecuronium bromide (Norcuron) was infused rapidly to induce paralysis, which was maintained by a continuous infusion of Norcuron ( $100 \mu \mathrm{g} / \mathrm{kg} / \mathrm{hr})$ in lactated Ringer's solution. The monkey was ventilated artificially at $30 \mathrm{strokes} / \mathrm{min}$ at a tidal volume adjusted to keep the end-tidal $\mathrm{CO}_{2}$ close to $33 \mathrm{~mm} \mathrm{Hg}$. Throughout the experiment the level of anesthesia was monitored by the elec- troencephalogram, which was maintained in a state consistent with light sleep by supplementary doses of Nembutal or (in later experiments) a continuous infusion of Sufenta (initially $2 \mu \mathrm{g} / \mathrm{kg} / \mathrm{hr}$, adjusted as necessary). The electrocardiogram was monitored as an indicator of the animal's general health. A heating blanket controlled by a subscapular thermistor prevented body temperature falling below $37^{\circ} \mathrm{C}$.

Pupils were dilated with atropine sulfate and the corneas were protected with opaque contact lenses that contained artificial pupils 2.5 $\mathrm{mm}$ in diamctcr. Supplementary lenses were usually required to focus stimuli on the retina; these were chosen during preliminary observations by finding the power that permitted the resolution of the highest spatial frequencies. The lenses were removed approximately every $12 \mathrm{hr}$ and the eyes were washed with saline. If there were signs of corneal clouding, the eyes were closed for an hour or more before the lenses were replaced. At the beginning of the experiment, and each time the eyes were examined, the foveal reflex was found and projected by reversed ophthalmoscopy onto a tangent screen $1.6 \mathrm{~m}$ in front of the animal.

Action potentials were recorded with glass-insulated tungsten microelectrodes (Levick, 1972), positioned with a stepping-motor driven micrometer. In order to maximize the number of cells studied in each cortical layer, long tangential penetrations were made through striate cortex containing the representation of the central $3^{\circ}$. An electrolytic lcsion was made at the end of each penetration, and further lesions were made at irregular intervals during withdrawal of the electrode. This helped identify penetrations unambiguously. At the end of the experiment the monkey was perfused through the heart with $0.9 \%$ saline in neutral phosphate buffer, followed by a solution of $1.25 \%$ paraformaldehyde and $2.5 \%$ glutaraldehyde. The brain was blocked and sunk in $10 \%$ then $30 \%$ sucrose, after which it was frozen and cut in $50 \mu \mathrm{m}$ sections. Alternate sections were stained for Nissl substance and cytochrome oxidase.

Visual stimuli. An important feature of the present experiments was that neurons be studied in a stable state of adaptation. All stimuli were therefore defined by spatiotemporal modulation about some steady chromaticity and luminance. Figure $1 A$ shows the 3-dimensional color space in which these modulations are represented. Along one axis of this space (the "constant B" axis) modulation brings about no change in the excitation of short-wavelength-sensitive (B) cones while causing the signals in the long-wavelength-sensitive $(R)$ and middle-wavelengthsensitive (G) cones to covary, so as to keep their sum constant. Along the second ("constant R,G") axis, modulation brings about no change in the excitation of $R$ and $G$ cones. A plane in which only chromaticity varies is defined by these axes; their intersection defines the adaptation point (typically white), about which all stimuli are modulated. The third (achromatic) axis of the color space is one of radiance variation: Signals from all 3 classes of cone vary in proportion along this axis.

The 2 chromatic axes of this color space correspond to the directions along which modulation is especially effective for the 2 classes of chromatically opponent neuron found in parvocellular layers of l.g.n. (Derrington et al., 1984): Neurons that receive opposed inputs from $R$ and $G$ cones respond well to modulation along the constant $B$ axis and are blind to modulation along the constant $R, G$ axis; neurons that receive inputs from $B$ cones opposed to some combined signal from $R$ and $G$ cones respond well to modulation along the constant $R, G$ axis and are blind to modulation along the constant $B$ axis. In all the experiments described here, the stimuli were spatially uniform fields modulated in time along some vector through the adaptation point or were moving 1-dimensional grating patterns defined by modulation along some vector through the same point. A direction in the color space is defined by an azimuth $(\phi)$ and an elevation $(\theta)$. Both terms are explained in the legend to Figure 1.

Stimuli were generated by a PDP 11/60 computer and delivered to a Tektronix 690SR color television monitor by an Adage 3000 framebuffer and controller. For the present experiments the visible display was refreshed at $120 \mathrm{framcs} / \mathrm{scc}$ (interlaccd) and measured $37 \mathrm{~cm}(600$ pixels) horizontally by $27.5 \mathrm{~cm}$ ( 450 pixels) vertically. The monitor was placed $5.4 \mathrm{~m}$ from the animal, at which distance the screen subtended $2.8^{\circ} \times 3.4^{\circ}$. The frame-buffer controller could display arbitrary 2-dimensional stimuli; in the present experiments it was used to produce patches of grating lying in the required orientation, at the appropriate place on the television screen. The spatial waveform was held in the frame-buffer with an accuracy of 8 bits, and the chromaticity and luminance of the pattern were determined by using the 8-bit value of each point on the waveform as an index to 3 tables that contained intensity values for the $\mathrm{R}, \mathrm{G}$, and $\mathrm{B}$ signals delivered to the monitor. Values in 
these tables, which were held to an accuracy of 10 bits, could be rewritten during the time taken for frame fly-back on the monitor, so chromaticity and luminance could be modulated at $60 \mathrm{~Hz}$. The nonlinear relationships between applied voltages and phosphor luminances were corrected by compensating nonlinearities in the 3 tables.

The projections of the constant $B$ and constant $R, G$ axes are drawn on the chromaticity diagram shown in Figure $1 B$; their intersection $(x$ $=0.311, y=0.336$ ) defines the adaptation point. This was chosen to be close to equal energy white, without unduly restricting the maximum luminance available from each phosphor. The projection of a true constant $B$ axis should intersect the chromaticity diagram at $x=1.0, y=$ 0 . However, it was computationally convenient to use an axis along which only the $R$ and $G$ primaries were modulated. This resulted in our constant $\mathrm{B}$ axis passing through the coordinates $x=1.10, y=0$, and in the color space of Figure $1 A$ lying $0.4^{\circ}$ from its correct position. In the remainder of this paper, we ignore the mislocation of the axis. The constant R,G axis (a tritanopic confusion line) is defined by a line connecting the adaptation point and the tritanopic copunctal point (Walraven, 1974). The space-time average luminance was $120 \mathrm{~cd} / \mathrm{m}^{2}$.

Unit modulation along the luminance axis produced a Michelson contrast of 1.0. There is no agreed means of calculating the modulation of a heterochromatic grating. The maximum attainable modulation along the constant B axis through the adaptation point gave rise to Michelson contrasts of 0.074 to the $R$ cones, 0.140 to the $G$ cones, and 0.018 to the $B$ cones; the maximum attainable modulation of chromaticity along the constant $R, G$ axis gave rise to the following modulations of quantum catches: $R, 0.00 ; \mathrm{G}, 0.00 ; \mathrm{B}, 0.84$. As a practical device for calculating $\theta$ and $\phi$, unit modulation along the constant $\mathrm{R}, \mathrm{G}$ axis was defined as the maximum attainable; unit modulation along the constant $B$ axis was 0.32 of the maximum attainable.

Examination of receptive fields. All receptive fields lay within $3^{\circ}$ of the center of the fovea. When a neuron was first isolated, the receptive field of the dominant eye was marked on the tangent screen (the nondominant eye was covered). The position of the receptive field was then marked by a laser beam reflected off a coverslip placed just below the eye; this beam was subsequently reflected off a front surface mirror on to the center of the television screen.

Since cortical neurons are often selective for several attributes of a stimulus, and since most cortical neurons respond to achromatic stimuli, our strategy was to characterize the receptive field with achromatic stimuli and then, with stimuli of the favored configuration, vary the chromatic properties for best response. A preliminary examination was made with a small patch of achromatic grating, whose spatial frequency, orientation, length, width, and position could be continuously varied. If the preliminary exploration of the receptive field indicated that a neuron was selective for the spatial frequency or orientation of an achromatic grating, the spatial-frequency selectivity and orientational selectivity were examined quantitatively. To measure spatial selectivity, the neuron was driven by gratings of the (estimated) optimal orientation at a range of spatial frequencies covering the frequency estimated to be optimal. We were particularly interested in responses to low and middle spatial frequencies, so our measurements seldom explored the highest frequencies to which cells responded. The optimal orientation was then found using gratings of the preferred spatial frequency at a range of orientations centered on the one thought to be optimal. Some neurons responded so poorly to achromatic stimuli that their receptive fields could not be characterized adequately. In such cases, the initial exploration of the receptive field was made with colored stimuli to which the neuron did respond.

After the optimal spatial configuration had been established, the optimal chromatic stimulus was sought by systematic examination of responses to stimuli of this configuration modulated in different directions about the adaptation point. Such an approach allows us very quickly to restrict the domain to be explored, but a potential weakness is that if the spatial and chromatic properties of the receptive field are not separable (i.e., the preferred spatial frequency depends upon the chromatic properties of the stimulus), we may be unable to find the optimal

$\overline{\text { Figure 2. A, Spatial frequency characteristic of a nonoriented neuron }}$ in layer VI. Open circles show the amplitude of the first-harmonic component of response to movement of an achromatic grating of unit contrast; filled circles show the amplitude of the same component of response to the maximum attainable modulation along the constant $\mathrm{B}$
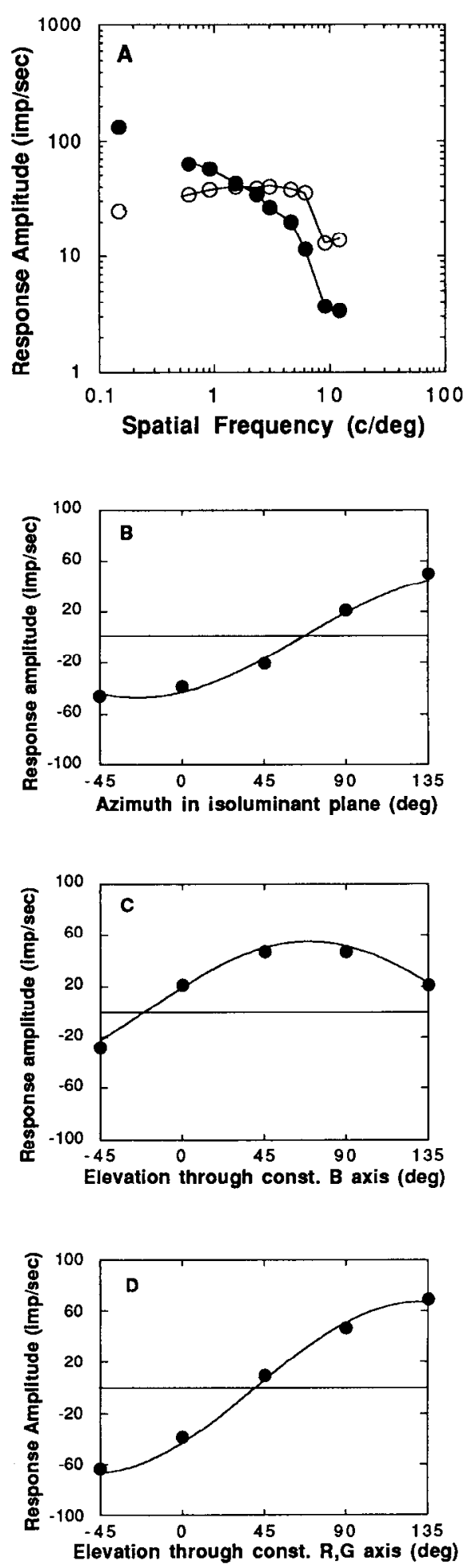

axis $\left(\phi=0-180^{\circ}\right)$. The isolated points at the left show the amplitudes of responses to modulation of a spatially uniform field. $B$, Amplitude of first-harmonic component discharge to temporal modulation of a spatially uniform field along selected vectors in the isoluminant plane of the color spacc. Amplitudes arc normalized for stimuli of unit contrast. $C$, Responses to modulation along vectors in the vertical plane defined by the achromatic axis and the constant $B$ axis. $D$, Responses to modulation along vectors in the vertical plane defined by the achromatic axis and the constant $R, G$ axis. Smooth curves show the bestfitting solution to Eq. 1, obtained from concurrent analysis of all points. For this neuron $\theta_{m}=47.2^{\circ}$ and $\phi_{m}=156^{\circ}$. 


\section{$\theta$}

$90^{\circ}$

$45^{\circ}$
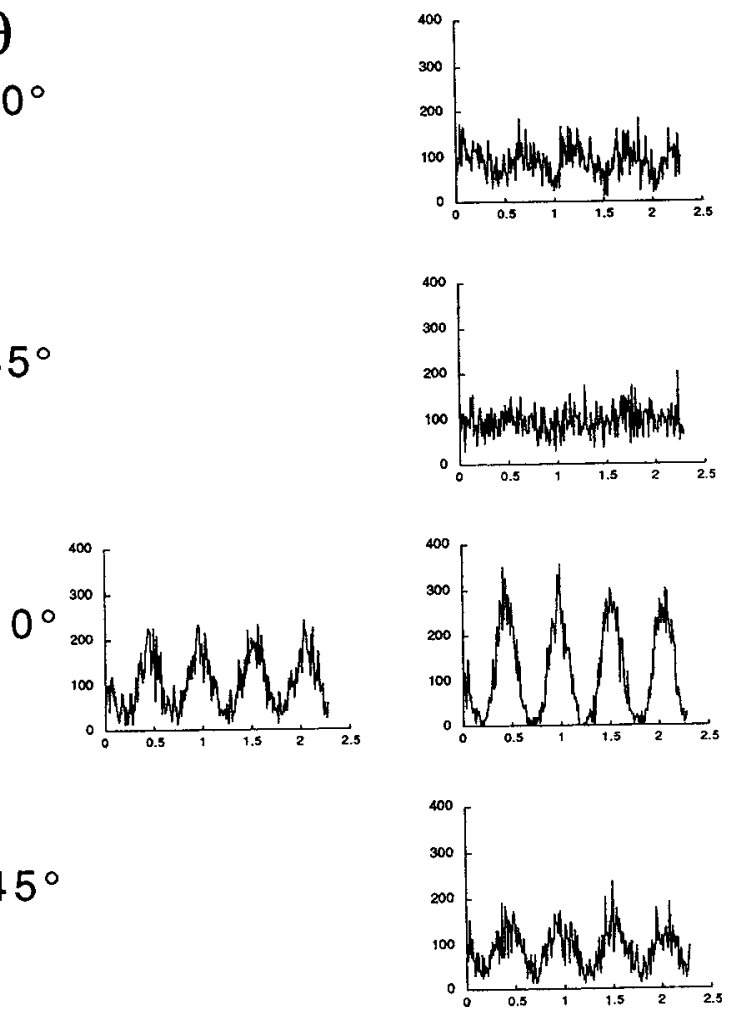
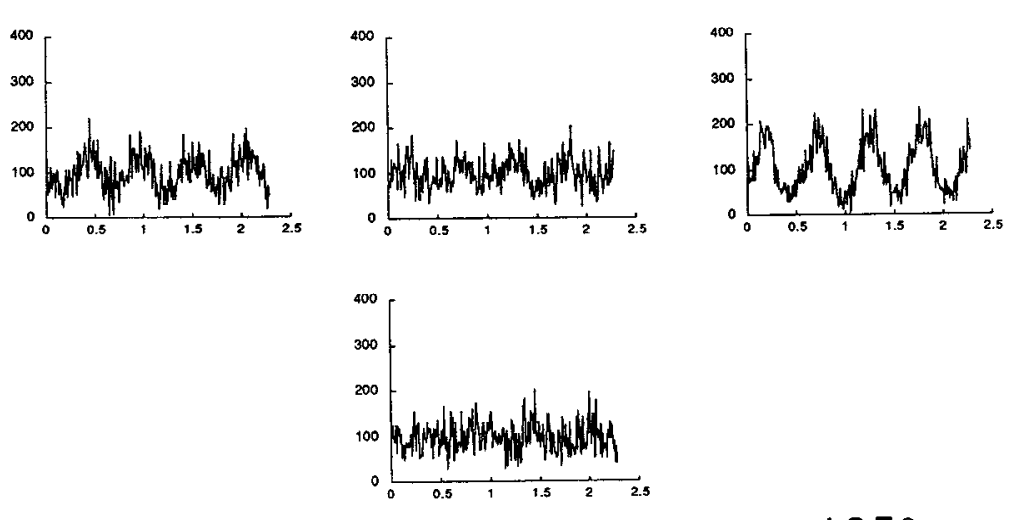

$45^{\circ}$
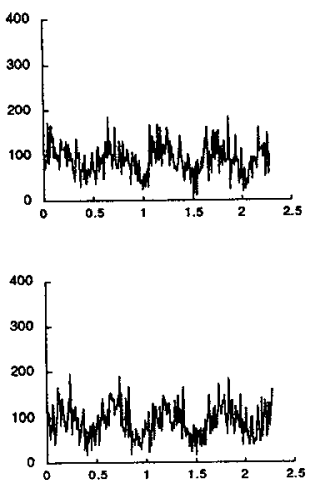

$90^{\circ}$

Figure 3. Histograms showing, for the cell of Figure 2, the pattern of average discharge to a spatially uniform field modulated at $1.68 \mathrm{~Hz}$ along different vectors in the 3 principal planes of the color space. Rows contain histograms of responses to modulation along vectors of constant elevation (the long row for $\theta=0^{\circ}$, the isoluminant plane) and columns contain histograms of responses to modulation along vectors of constant azimuth. The amplitude of modulation was fixed at 0.5 , except for gratings modulated along vectors in the isoluminant plane, where it was set to the maximum attainable. Each histogram contains the response to 20 presentations of the stimulus. Calibration: ordinate, $100 \mathrm{imp} / \mathrm{sec}$; abscissa, 0.5 sec. Bin width $8.4 \mathrm{msec}$.

stimulus. If the preferred direction of modulation lay far from the achromatic axis, the spatial and orientational selectivities were usually examined again using gratings modulated in the preferred direction.

In all experiments, stimuli were modulated sinusoidally in time at $1.86 \mathrm{~Hz}$ or moved steadily across the receptive field at the same rate. The different stimuli required in a single experiment (e.g., to measure orientational selectivity) were each presented for $2.28 \mathrm{sec}$ ( 4.25 cycles), and were randomly interleaved. A blank interval of $1 \mathrm{sec}$ was inserted between successive stimuli. In most experiments, each stimulus was presented 20 times.

Classification of cells. During the preliminary examination of its receptive field an orientation-selective neuron was classified as "nonoriented," "simple," or "complex." This distinction was later drawn more firmly by analysis of the responses of neurons to moving sinusoidal gratings. Following DeValois et al. (1982), we called cells "simple" when the ratio of amplitudes of the fundamental component of response to that of the unmodulated component was greater than 1. Later in this paper, we analyze the chromatic properties of cells in 2 different ways, depending upon whether the neurons summed signals from cones linearly. All simple cells and nearly all nonoriented cells were subjected to the linear analysis, complex cells, and the few nonoriented cells that gave second harmonic responses, to the nonlinear one.

Analysis of response. Action potentials were amplified and used to trigger rectangular pulses that were recorded by the computer. Pulse counts were accumulated in an array of bins (each $8.4 \mathrm{msec}$ wide) indexed by the phase of the stimulus. The amplitudes and phases of component responses at different temporal frequencies were extracted by taking the Fourier transform of the array. Each sample contained the response to at least 4 cycles of stimulus modulation, and to avoid contamination by transients, the early part of the response was excluded from the analysis. A histogram showing average discharge rate against time was also constructed from the recorded action potentials.

\section{Results}

Our goals were to characterize the chromatic sensitivities of neurons and to establish how they transform signals from l.g.n. The methods introduced in our earlier work (Derrington et al., 1984) permit a full description of the chromatic properties of neurons, but only if cells sum their inputs linearly. We begin by showing that these methods adequately characterize the chromatic properties of simple cells and cells that are not orientationselective, then show how an extension of them copes with the behavior of complex cells.

Quantitative observations were made on 305 neurons in striate cortex; 2 penetrations extended into $\mathrm{V} 2$, in which 6 neurons were studied. We observed a wide range of properties in the population examined and have illustrated this with examples.

\section{Neurons that sum signals linearly \\ Nonoriented cells}

The macaque's striate cortex contains many neurons that have linearly summing receptive fields, are monocularly driven, lack orientation specificity, and generally respond well to gratings of low spatial frequency. Hubel and Wiesel (1968) found cells like this to be common in layer IV, and we often encountered them; indeed, in several experiments we sought them because many have chromatically interesting properties. Although we have no evidence that these "nonoriented" cells differ qualitatively from 

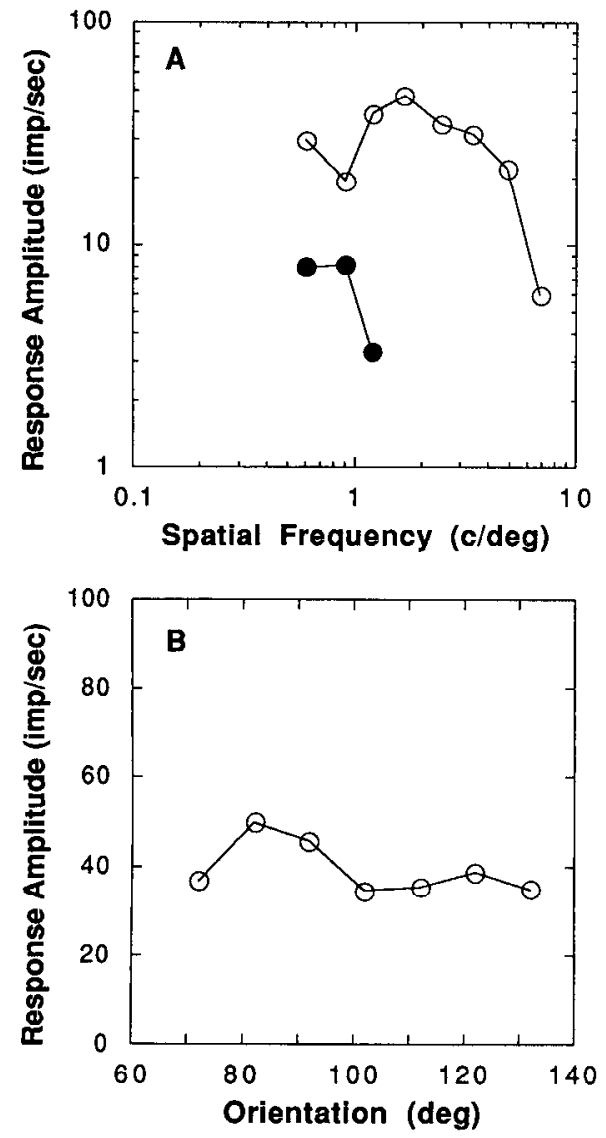
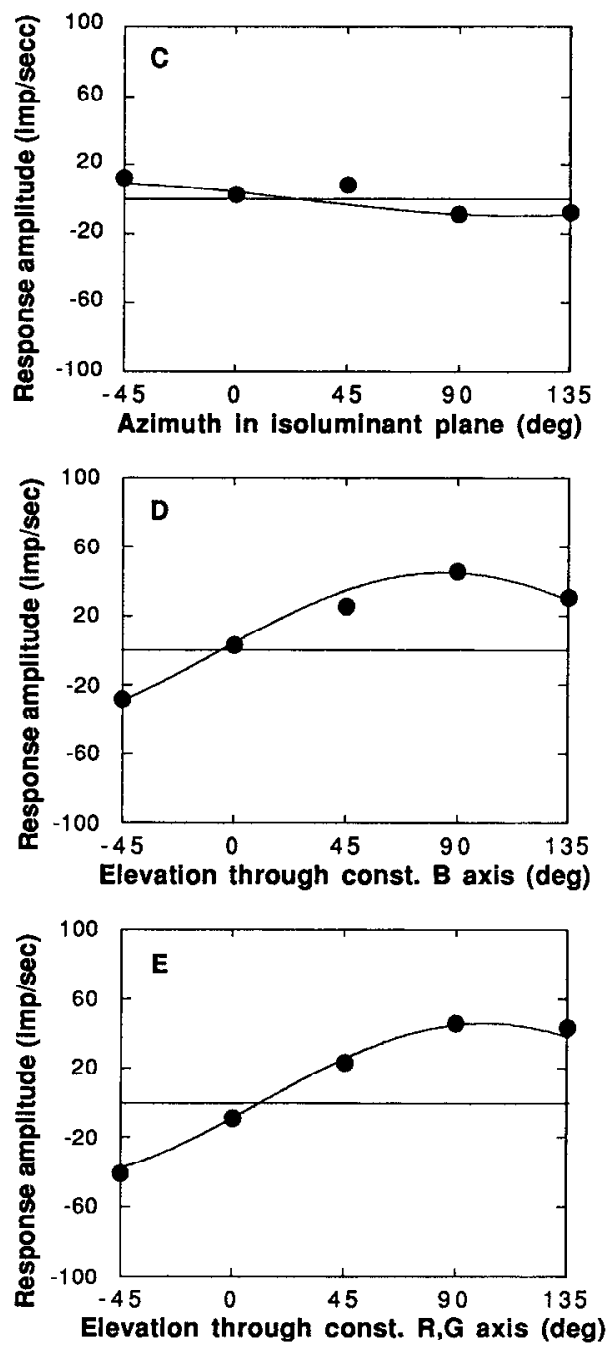

Figure 4. $A$, Spatial frequency characteristic of a linearly summing neuron lying in a cytochrome oxidase blob in layer II/III. Open circles, achromatic grating; filled circles, grating modulated in the isoluminant plane along the constant $\mathrm{R}, \mathrm{G}$ axis $\left(\phi=90^{\circ}-270^{\circ}\right)$. B, Orientation selectivity for achromatic gratings of optimal spatial frequency. $C-E$, Amplitude of first-harmonic component of discharge to gratings modulated along selected vectors in the 3 principal planes of the color space. Conventions as for Figure 2. $\theta_{m}=-73.7^{\circ}, \phi_{m}=279^{\circ}$. orientation-selective simple cells, we have followed Hubel and Wiesel in distinguishing them informally.

The open circles in Figure $2 A$ show, for a monocularly driven cell in layer VI, the spatial selectivity measured with moving achromatic gratings. The optimum direction of modulation in color space was then sought by measuring responses to gratings (of the spatial frequency found to be optimal for achromatic gratings) modulated along vectors spaced $45^{\circ}$ apart in the 3 principal planes of the color space (i.e., planes defined by the intersection of 2 cardinal axes). Figure 3 shows, for the cell whose spatial properties are illustrated in Figure $2 A$, how the response to the grating varied with direction of modulation in the 3 principal planes passing through the adaptation point. The amplitude of the modulated response varied regularly with vector rotation in each plane, and underwent a reversal in phase at some point. Figure 2, $B-D$, shows, for each of these planes, how the amplitude of the Fourier component of response at the frequency of stimulus modulation varied with the direction of modulation. In this and subsequent figures, positive and negative amplitudes are used to represent responses of opposite phases.

If a cell combines cone signals linearly, and responds linearly to the sum of these signals, it can be shown (Derrington et al., 1984) that there will be one plane (the "null" plane) containing the adaptation point within which modulation brings about no response from the cell, and orthogonal to this an axis passing through the adaptation point along which modulation maximally excites the cell. The amplitude $\mathrm{R}$ of the response to modulation along a vector of elevation $\theta$ and azimuth $\phi$ is given by the dot product of the stimulus vector and a vector in the direction of best response, namely,

$$
R=K\left[\sin \theta \sin \theta_{m}+\cos \theta \cos \theta_{m} \cos \left(\phi-\phi_{m}\right)\right],
$$

where $K$ is a scale factor, $\theta_{m}$ the elevation of the vector of maximal response, and $\phi_{m}$ the azimuth of the vector of maximal response. Equation 1 simply reflects the fact that the response to any stimulus is proportional to its projection along the direction of maximal response $\left(\theta_{m}, \phi_{m}\right)$. $R$ therefore varies sinusoidally with vector rotation in any plane that contains the adaptation point; in the null plane, $R$ is zero. For a cell whose behavior is described by Eq. $1, K, \theta_{m}$, and $\phi_{m}$ completely characterize its chromatic properties for the particular spatial and temporal conditions of the measurement. Derrington et al. (1984) took the parameters of the orthogonal null plane as the chromatic signature, but that signature does not express the direction of the vector of maximal response.

The minimizing routine STEPIT was applied to the data to derive a single set of values of $K, \theta_{m}$, and $\phi_{m}$ that best fitted the responses to stimuli modulated in all 3 planes. For the points plotted in Figure 2, $B-D$, this procedure yields values of $47.2^{\circ}$, 
Figure 5. A, Spatial frequency characteristic of a simple cell in an unknown layer. Measurements were made with an achromatic grating. The isolated point at the left shows the amplitude of response to modulation of a spatially uniform field. The cell did not respond to chromatically modulated gratings. $B$, Measurements of orientation selectivity for achromatic gratings of optimal spatial frequency. $C-E$, Amplitude of first-harmonic component of discharge to gratings of $1.4 \mathrm{c} / \mathrm{deg}$ modulated along selected vectors in the 3 principal planes of the color space. Conventions as for Figure 2. $\theta_{m}=-87.2^{\circ}, \phi_{m}=116.7^{\circ}$.
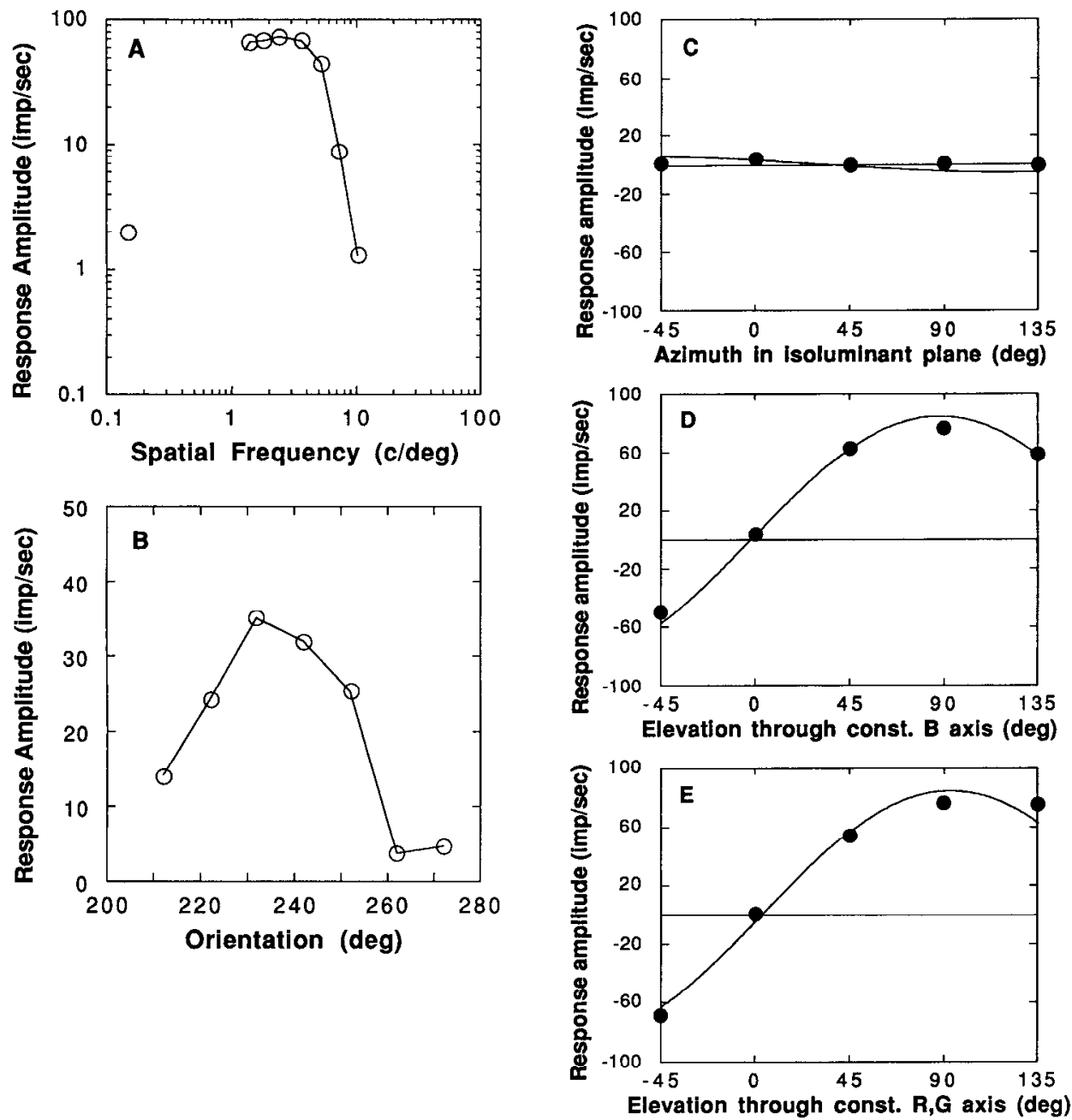

$156^{\circ}$, and 69.4 for $\theta_{m}, \phi_{m}$, and $K$, respectively, from which the solid lines in the figure are computed. The good fit of the sinusoid validates our assumption of linearity. The relationship between contrast and amplitude of response was measured directly for most cells. For many, the response varied linearly with contrast at low amplitudes but saturated at high amplitudes, and this can be seen in some following figures (e.g., Fig. 7); for some cells, the relationship was clearly nonlinear over its whole range (see Sclar et al., 1990), and the fit of Eq. 1 could have been improved by making allowance for this.

The open circles in Figure $4 A$ show the spatial selectivity (measured with achromatic gratings) of a unit in a cytochromeoxidase "blob" in layer II/III. This cell was weakly orientation selective (Fig. 4B), and because it responded well to gratings of low spatial frequency, its chromatic preferences were examined using a grating of $1.4 \mathrm{c} / \mathrm{deg}$ modulated in different directions through the adaptation point. Figure 4, $C-E$ shows how the amplitude of response varied with vector rotation in the 3 principal planes of the color space. The fit of the linear model is indicated by the solid lines; the best-fitting values of $\theta_{m}$ and $\phi_{m}$ were $73.7^{\circ}$ and $279^{\circ}$, respectively.

\section{Simple cells}

The chromatic properties of simple cells were also well characterized by Eq. 1: the open circles in Figure 5, $A$ and $B$, shows, for a cell in an unknown layer, how the amplitude of response varied with the spatial frequency and orientation of an achromatic grating; Figure 5, $C-E$, shows how the amplitude of response to a stimulus of optimal spatial frequency varied with direction of modulation about the adaptation point. The preferred direction of modulation, calculated from the best-fitting solution to Eq. 1, was $\theta_{m}=-87.2^{\circ}$ and $\phi_{m}=116.7^{\circ}$. Figure 6 shows the results of similar measurements made on a simple cell that lay in layer IVA. This cell responded well to chromatically modulated gratings of low spatial frequency.

The neurons whose behaviors are shown in Figures 2-6 had $\theta_{m}$ spanning the range of possible values: The simple cell in Figure 5 was most sensitive to modulation along a vector lying close to the achromatic axis, while the unit of Figure 6 was most sensitive to stimuli modulated along a vector lying close to the isoluminant plane. These chromatic properties depended somewhat upon the spatial frequency of the grating used in the measurement, a point to which we return later.

\section{Neurons that sum signals nonlinearly}

Equation 1 characterizes the chromatic properties of cells that sum signals linearly, but it does not adequately describe the behavior of 2 sorts of nonlinear cell distinguished in the present work. 

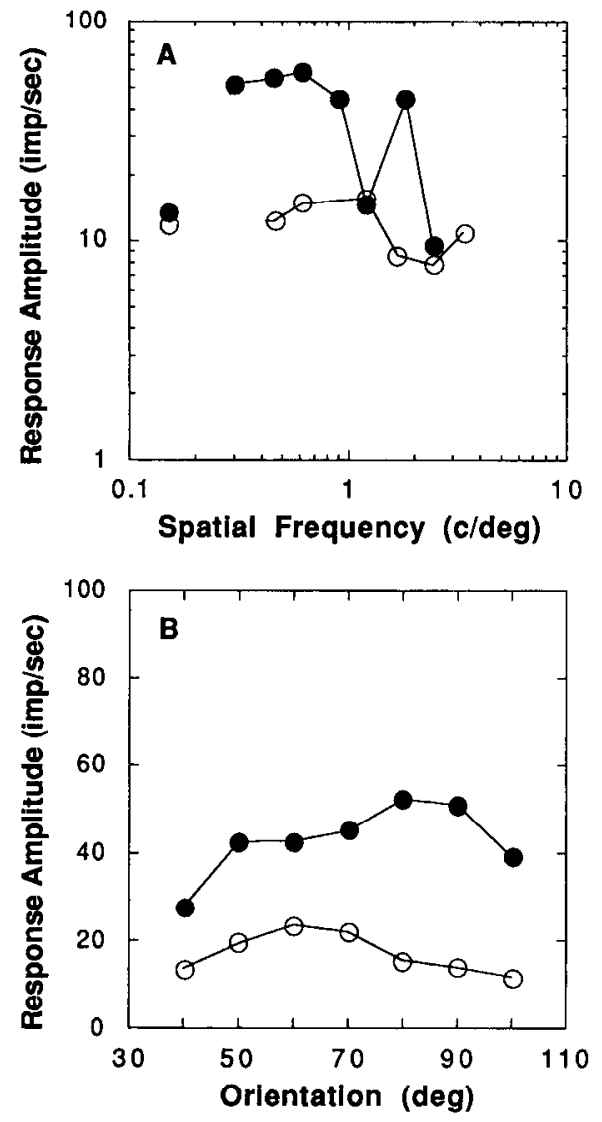

\section{Complex cells}

The most prevalent nonlinearity in cortical neurons is typified by the behavior of a complex cell, which generally responds with an unmodulated discharge to movement of a grating across the receptive field (Movshon et al., 1978; DeValois et al., 1982a). We encountered some complex cells (particularly in layer II/ III) that showed little selectivity for orientation; in what follows, we have not distinguished these from the more commonly encountered ones that show pronounced orientation selectivity.

Figure $7 A$ shows how the amplitude of the unmodulated component of response of a complex cell in layer II/III (outside a region rich in cytochrome oxidase) varied with the spatial frequency of a moving achromatic grating of optimal orientation; in this case, as in many others, we collected only enough information to identify the peak of the curve and to characterize its low-frequency limb. The orientational selectivity is shown in Figure $7 B$. Figure 8 shows the responses of the same cell to a moving grating of $3.6 \mathrm{c} / \mathrm{deg}$ modulated along different vectors in the 3 principal planes of the color space (the cell responded to no modulation of a spatially uniform field). The major component of the response is a substantial unmodulated increase in discharge, and this nonlinearity cannot be accommodated by Eq. 1.
Figure 6. A, Spatial frequency characteristic of a linearly summing neuron in layer IVA. Open circles, achromatic grating; filled circles, grating modulated in the isoluminant plane along the constant $\mathrm{R}, \mathrm{G}$ axis $\left(\phi=90^{\circ}-270^{\circ}\right)$. The isolated points on the left show the amplitudes of responses to modulation of a spatially uniform field. $B$, Orientation selectivity of the cell measured with gratings of optimal spatial frequency. Orientations are counterclockwise from 3 o'clock. $C-E$, Amplitude of first-harmonic component of discharge to gratings modulated along selected vectors in the 3 principal planes of the color space. Conventions as for Figure 2. $\theta_{m}$ $=17.6^{\circ}, \phi_{m}=89.2^{\circ}$.
The simplest and most complete account of the behavior of the complex cell in cat is offered by Spitzer and Hochstein (1985a, b), who suggest that signals from linearly summing elements distributed throughout the receptive field are half-wave rectified before being combined linearly. We have found this model helpful in characterizing the responses of complex cells to stimuli modulated along different vectors in color space. When the constituent mechanisms are excited in phase (for example, by temporal modulation of a spatially uniform field), the response of the cell will be half-wave rectified, and its amplitude will vary sinusoidally with vector direction as per Eq. 1. (The amplitude of the response is taken to be negative when the phase changes by $180^{\circ}$.) However, if the elements are excited in random temporal phase, as is likely to occur when a grating moves steadily across the receptive field, there will be a continuous elevation of discharge whose amplitude varies as a full-wave rectified sinusoidal function of vector direction. This behavior can be described by the following equation, which incorporates the linear color model used earlier to characterize nonoriented and simple cells:

$$
R=O+K\left|\left[\sin \theta \sin \theta_{m}+\cos \theta \cos \theta_{m} \cos \left(\phi-\phi_{m}\right)\right]\right|,
$$

where $O$ is the maintained discharge in the absence of any stimulus. 
Figure 7. A, Spatial frequency characteristic of a complex cell in layer II/ 1II. Measurements made with achromatic gratings. $B$, Orientation selectivity for achromatic gratings of optimal spatial frequency. $C$, Amplitude of unmodulated component of discharge to a moving grating of $3.6 \mathrm{c} / \mathrm{deg}$, modulated along selected vectors in the isoluminant plane of the color space. Amplitudes are normalized for stimuli of unit contrast. $D$, Responses to modulation along vectors in the vertical plane defined by the achromatic axis and the constant $\mathrm{B}$ axis. $E$, Responses to modulation along vectors in the vertical plane defined by the achromatic axis and the constant $R, G$ axis. Smooth curves show best-fitting solution to Eq. 2. For this neuron, $\theta_{m}=87.2^{\circ}$ and $\phi_{m}$ $=360^{\circ}$.
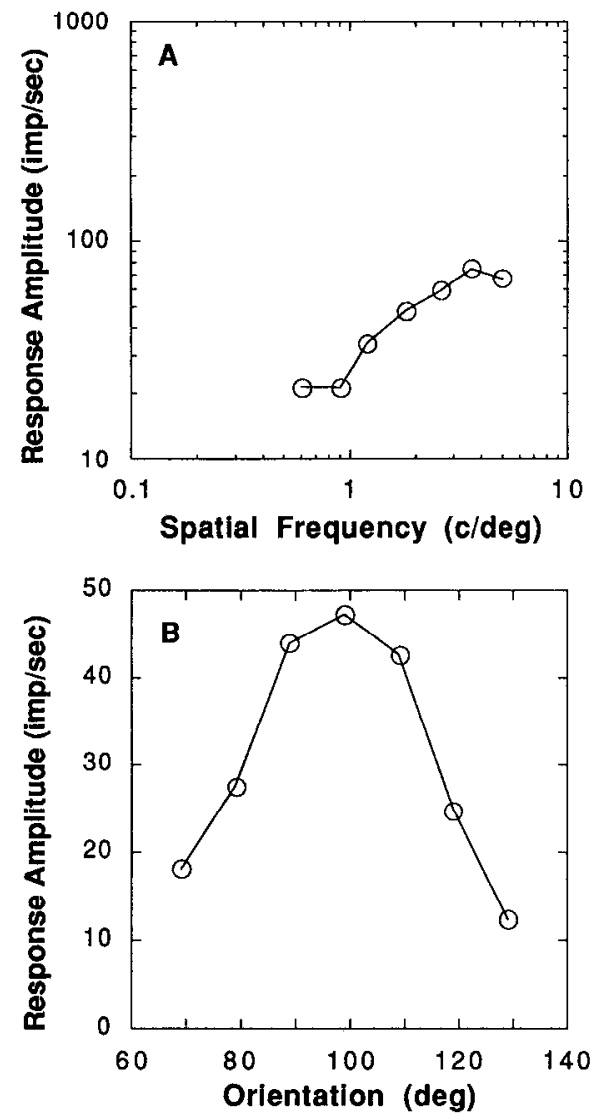
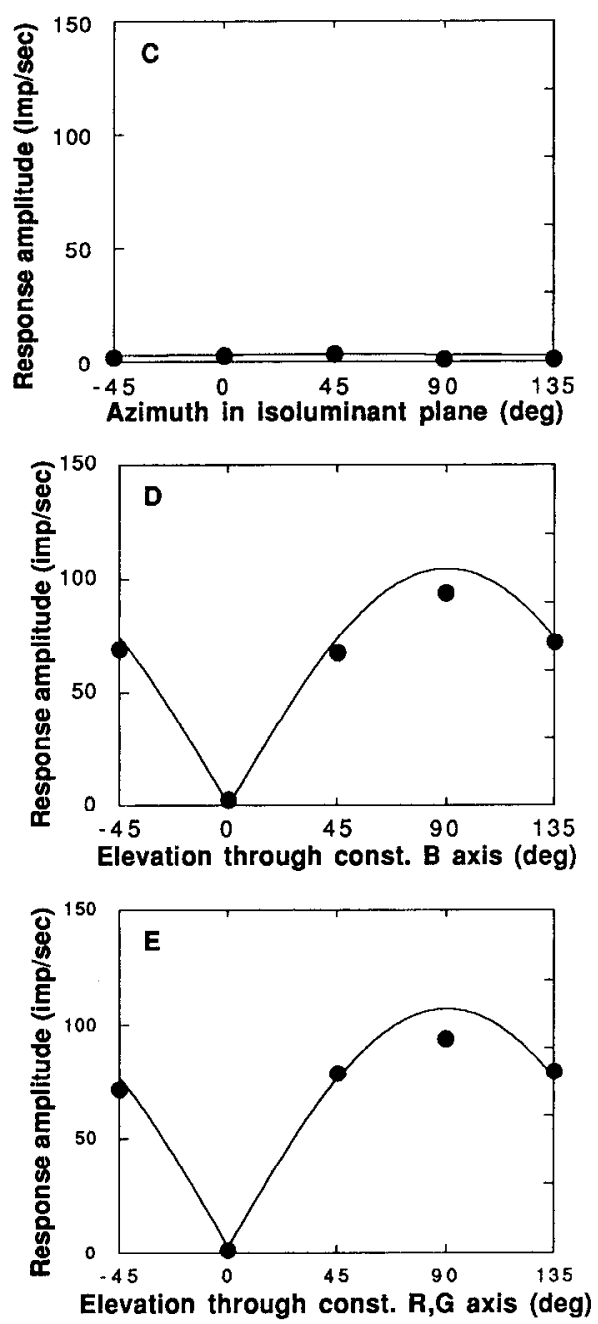

The 3 panels in Figure 7, $C-E$, show, for the responses depicted in Figure 8, how the average discharge rate varied with the direction in color space along which the grating was modulated. The solid lines show the best-fitting solution to Eq. 2 obtained by the minimizing routine STEPIT. As before, a single solution was computed from the responses to modulation in all 3 planes. This neuron responded best to stimulus modulation along the achromatic axis.

Figure $9, A$ and $B$, shows, respectively, the spatial and orientational selectivities of a second complex cell that lay within layer II/III, and Figure 9, $C-E$, shows, for a grating of the optimal spatial frequency and orientation, how the amplitude of the unmodulated component of discharge varied with the direction in color space along which the grating was modulated. Like the neuron whose behavior is shown in Figures 7 and 8 , it responded to no modulation of a spatially uniform field. The solid lines show the solution to Eq. 2, which evidently provides a useful means of characterizing the chromatic preferences of complex cells.

\section{Neurons sharply tuned for color}

Six neurons were highly selective for chromaticity. All gave wellmodulated responses to chromatic modulation of a spatially uniform field. To this extent, their behavior resembled that of the linear neurons whose chromatic selectivities are character- ized by Eq. 1. However, their selectivities for chromatic modulation were sharper than expected from Eq. 1, which described their behavior poorly. The responses of the most distinctive cell (from an unidentified layer) are shown in Figure 10. The other cells that behaved similarly were found in layers II/III, IVB, and VI. We do not yet have an adequate account of the mechanisms that produce this selectivity, but it may involve a linear subtractive interaction between broadly tuned mechanisms, followed by a threshold. Because we could have missed some cells with tuning substantially sharper than illustrated in Figure 10, narrowly tuned cells may be more common than our small sample suggests.

\section{Distribution of chromatic properties}

Figure $11, A-C$, shows, respectively, the distributions of $\theta_{m}$ and $\phi_{m}$ for linear nonoriented, simple, and complex cells, derived from measurements made with spatially uniform fields modulated in time (nonoriented cells) or moving gratings of optimal orientation and spatial frequency (simple and complex cells). Figure $11 D$ shows (from Derrington et al., 1984) the corresponding distributions for neurons in the parvocellular layers of the l.g.n. (The scatter plots show absolute values of $\theta_{m}$; values of $\phi_{m}$ lying between $135^{\circ}$ and $325^{\circ}$ are represented as $\phi_{m}-180$.) The histograms above and to the right of each plot show the distributions of $\phi_{m}$ and $\theta_{m}$, respectively. Since $\theta_{m}$ and $\phi_{m}$ are 


\section{$\theta$}

$90^{\circ}$

$45^{\circ}$
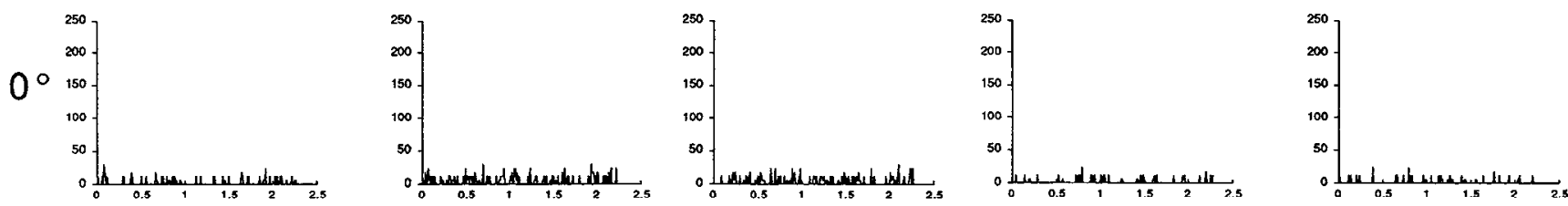

$-45^{\circ}$

$\phi \quad 315^{\circ}$
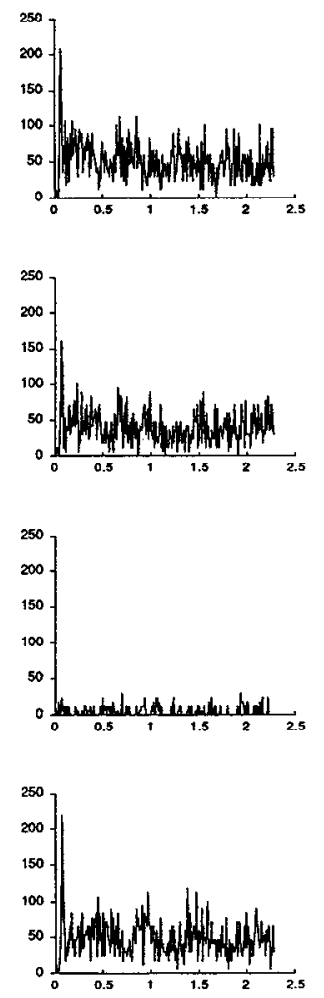

$0^{\circ}$
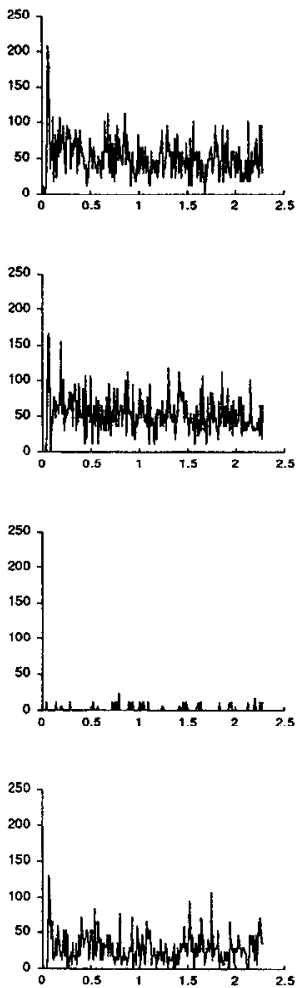

$90^{\circ}$ $135^{\circ}$

Figure 8. Histograms showing, for the neuron of Figure 7, the pattern of average discharge to moving gratings modulated along vectors in the 3 principal planes of the color space. Rows contain histograms of responses to gratings modulated along vectors of constant elevation (the long row for $\theta=0^{\circ}$, the isoluminant plane), and columns contain histograms of responses to gratings modulated along vectors of constant azimuth. The amplitude of modulation was fixed at 0.5 , except for gratings modulated along vectors in the isoluminant plane, where it was set to the maximum attainable. Histogram at lower right-hand corner shows discharge in the absence of any modulation. Each histogram contains the response to 20 presentations of the stimulus. Calibration: ordinate, $100 \mathrm{imp} / \mathrm{sec}$; abscissa, $0.5 \mathrm{sec}$; bin width, $8.4 \mathrm{msec}$.

represented in what is essentially a Mercator projection, $\phi_{m}$ becomes undefined as $\theta_{m}$ approaches $90^{\circ}$ (the achromatic axis). Cells with $\theta_{m}$ near $0^{\circ}$ respond best to stimuli that are purely chromatically modulated.

The population of linear nonoriented cells (Fig. 11A) contained many manifestly color-opponent neurons that preferred modulation along vectors close to the isoluminant plane, but the group as a whole was heterogeneous and had no clear R-G and B-R\&G subgroups of the kind found in parvocellular l.g.n. (Fig. 11D). If we consider only those nonoriented cells with $\theta_{m}$ $<45^{\circ}$, the distribution of $\phi_{m}$ becomes bimodal (solid bars in Fig. $11 A$ ), though the modes are less sharply defined than for neurons in l.g.n.

Figure 11, $B$ and $C$, shows that both simple and complex cells prefer stimuli modulated along vectors close to, but generally not along, the achromatic axis. It should be borne in mind that the chromatic properties of these neurons were established with gratings, so chromatic aberration will have acted to inflate the responses of cells to chromatically modulated gratings, and will thus have tended to lower $\theta_{m}$. We deal with this issue in the Discussion.

\section{Interpretation of $\theta_{\mathrm{m}}$ and $\phi_{\mathrm{m}}$}

$\theta_{m}$ and $\phi_{m}$ provide a functional description of the stimulus to which a neuron responds best: They provide an indication of what the preferred stimuli would look like and allow us to dis- cern classes of neurons that have distinct chromatic properties, if such exist. However, $\theta_{m}$ and $\phi_{m}$ depend on the units in which one calculates chromatic contrast, and this can be measured in different ways (our units are defined in Materials and Methods). It would therefore be helpful to have some more fundamental expression of the chromatic properties of neurons. A useful one is provided by the proportions in which a cell adds signals from the different classes of cones.

\section{Weights assigned to cone inputs}

The spectral sensitivities of the cones of Macaca fascicularis are now known precisely from the work of Baylor et al. (1987); those of $R$ and $G$ cones are indistinguishable from their human counterparts (Schnapf et al., 1987). If we assume that prereceptoral losses are the same for macaque and human, we can use Smith and Pokorny's (1975) fundamental sensitivities to calculate the quanta absorbed from any light by each class of cone.

When neurons in 1.g.n. and striate cortex are studied in a stable state of adaptation, the significant activity appears to be the change in discharge (modulated for linear nonoriented and simple cells, unmodulated for complex cells) brought about by modulating the stimulus. The average discharge rate appears to be unrelated to the level of illumination (the mean quantum catch in the cones)-indeed many cortical neurons have no maintained discharge at all-and the response of a neuron depends not on the absolute amplitude of stimulus modulation 
Figure 9. A, Spatial frequency characteristic of a complex cell in layer II/ III. Open circles, achromatic grating; filled circles, grating modulated in the isoluminant plane along the constant $\mathrm{R}, \mathrm{G}$ axis $\left(\phi=90^{\circ}-270^{\circ}\right)$. Left-hand points show responses to modulation of a spatially uniform field. $B$, Orientation selectivity for achromatic gratings of optimal spatial frequency. $C-E$, Amplitude of unmodulated component of discharge to a moving grating of 3.6 $\mathrm{c} / \mathrm{deg}$, modulated along selected vectors in the 3 principal planes of the color space. Conventions as for Figure 7. For this neuron, $\theta_{m}=90^{\circ}$ and $\phi_{m}=180^{\circ}$.
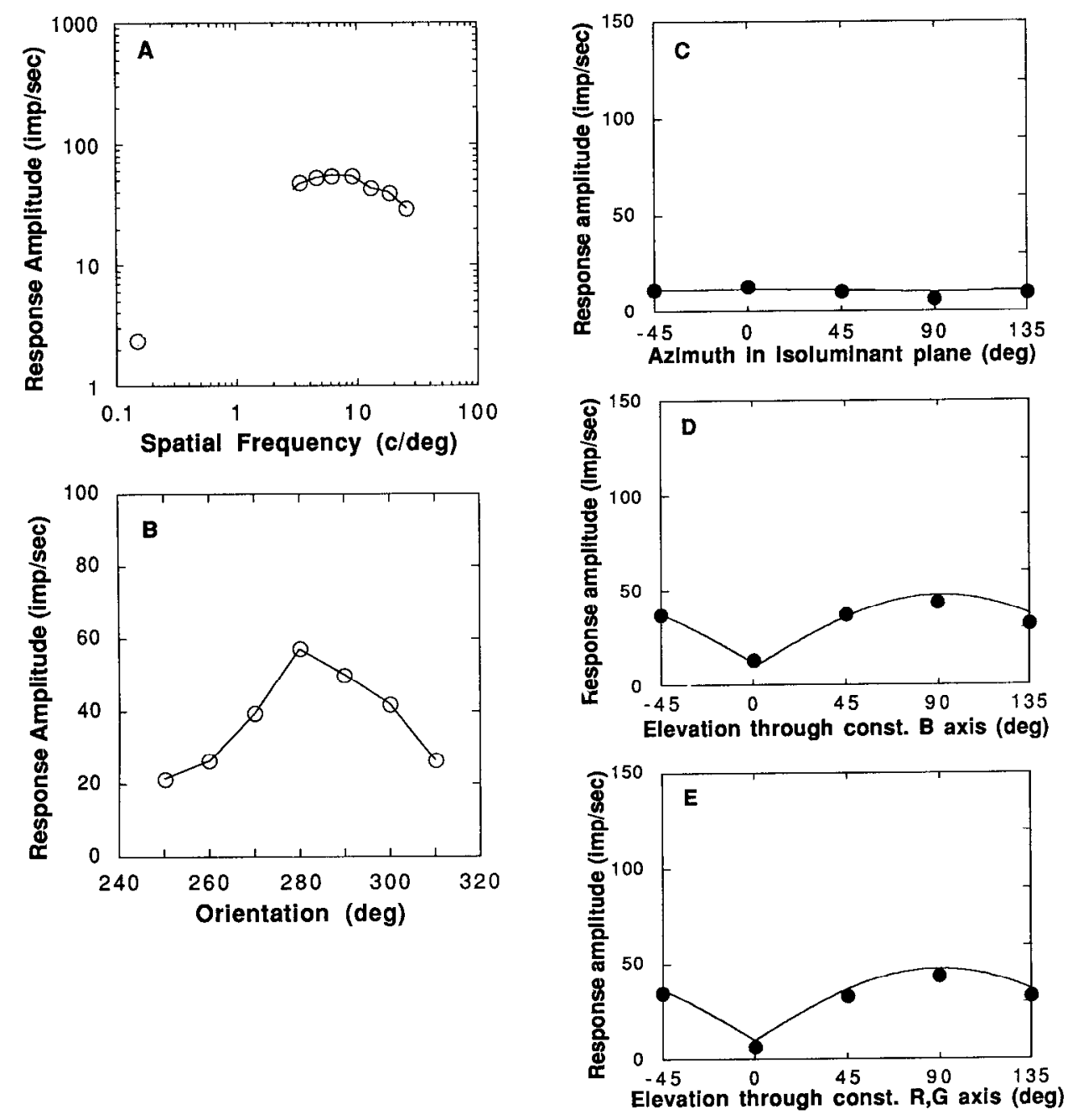

but on the relative amplitude of modulation (Michclson contrast), presumably as a result of light-adaptation in the retina. Most light-adaptation appears to occur in the separate cone pathways, before the opponent site (see Mollon, 1982, for a review), and in the following analysis we have therefore assumed that the input to the chromatically opponent site from any class of cone reflects the relative modulation of the quantum catch in that class of cone.

The amplitude of response of a simple cell or a linear nonoriented cell can be expressed as

$$
R=w_{r} M_{r}+w_{g} M_{g}+w_{b} M_{b},
$$

where $R$ is the amplitude of the fundamental component of response, $M_{r}, M_{g}$, and $M_{b}$ are the modulations of quantum catches in the $\mathbf{R}, \mathbf{G}$, and $\mathbf{B}$ cones respectively, and $w_{r}, w_{g}$, and $w_{b}$ are the weights with which the cell combines these modulated signals. $M_{r}, M_{g}$, and $M_{b}$ arc obtaincd by direct calculation or by transformation of Eq. 1 .

The relationship between $w_{r}, w_{g}$, and $w_{b}$ and $\theta_{m}, \phi_{m}$, and $K$ can be derived from Eq. 1 and Eq. 3 and is given by

$\left[\begin{array}{l}w_{r} \\ w_{g} \\ w_{b}\end{array}\right]\left[\begin{array}{ccc}\sin \theta_{r} & \sin \theta_{g} & \sin \theta_{b} \\ \cos \theta_{r} \cos \phi_{r} & \cos \theta_{g} \cos \phi_{g} & \cos \theta_{l} \cos \phi_{b} \\ \cos \theta_{r} \sin \phi_{r} & \cos \theta_{g} \sin \phi_{g} & \cos \theta_{b} \sin \phi_{b}\end{array}\right]=\left[\begin{array}{c}K \sin \theta_{m} \\ K \cos \theta_{m} \cos \phi_{m} \\ K \cos \theta_{m} \sin \phi_{m}\end{array}\right]$ where the constants $\theta_{r}, \theta_{g}$, and $\theta_{b}$ are the elevations, and $\phi_{r}, \phi_{g}$, and $\phi_{b}$ the azimuths of the directions of maximal response for the R, G, and B cones, respectively.

For complex cells, Eq. 3 is readily modified to represent the amplitude of the unmodulated increase in discharge brought about by the modulation of quantum catches. $w_{r}, w_{g}$, and $w_{b}$ can be estimated either by applying Eq. 4 to the estimated values of $\theta_{m}, \phi_{m}$, and $K$ or directly from the solution to Eq. 3 that best fits the results obtained from the experiment in which stimuli were modulated along a series of vectors passing through the adaptation point (e.g., right-hand panels in Figs. 2, 4-7, and 9). The values obtained for $w_{r}, w_{g}$, and $w_{b}$ depend on the absolute sensitivity of the cell, so to discount these variations in overall sensitivity, we obtain the relative weight for each cone class $\left(W_{,}\right.$, $W_{g}$, and $W_{b}$ ) by dividing each weight by the sum of the absolute weights attached to quantum catches in the 3 classes of cones, for example,

$$
W_{r}=\frac{w_{r}}{\left|w_{r}\right|+\left|w_{g}\right|+\left|w_{b}\right|} \text {. }
$$

Since relative weights sum to unity, they can all be represented on a plot of $W_{g}$ against $W_{r}$, in which $W_{b}$ is given directly. Figure 12 shows these plots for linear nonoriented cells $(A)$, simple cells $(B)$, complex cells $(C)$, and (from Derrington et al., 1984) neu- 

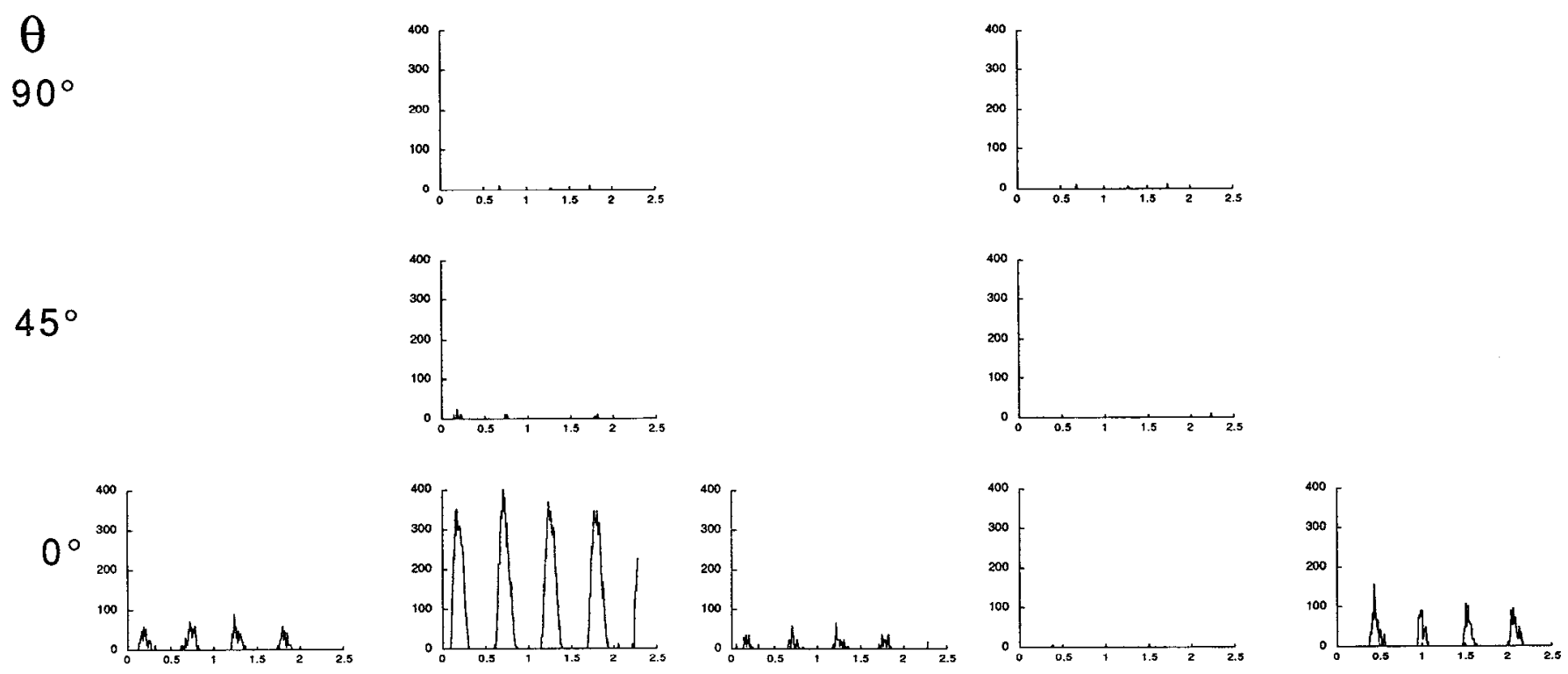

$-45^{\circ}$
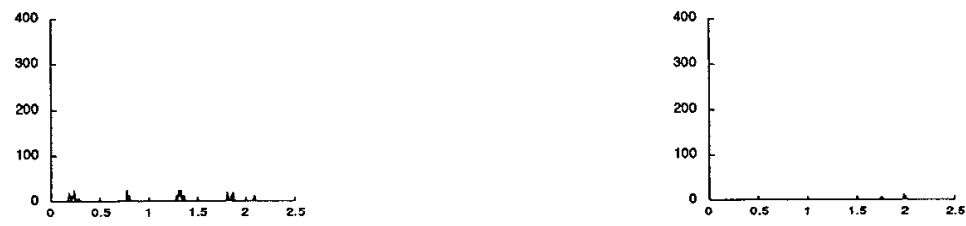

$\phi \quad 315^{\circ}$

$0^{\circ}$

$45^{\circ}$

$90^{\circ}$

$135^{\circ}$

Figure 10. Responses of a neuron sharply tuned for hue. Histograms show pattern of average discharge to modulation of a spatially uniform field along vectors in the 3 principal planes of the color space. Rows contain histograms of responses to gratings modulated along vectors of constant elevation (the long row for $\theta=0^{\circ}$, the isoluminant plane), and columns contain histograms of responses to gratings modulated along vectors of constant azimuth. The amplitude of modulation was fixed at 0.5 , except for gratings modulated along vectors in the isoluminant plane, where it was set to the maximum attainable. Each histogram contains the response to 20 presentations of the stimulus. Calibration: ordinate, 100 imp/sec; abscissa, $0.5 \mathrm{sec}$; bin width, $8.4 \mathrm{msec}$.

rons in parvocellular l.g.n. (D). Units that are driven by signals from $\mathrm{R}$ and $\mathrm{G}$ cones only are represented by points that lie on the diagonals connecting unit values on the axes; if a neuron receives opposed inputs from $R$ and $G$ cones, it is represented in the second or fourth quadrants; if a unit receives signals of the same polarity from $R$ and $G$ cones, it is represented in the first or third quadrants. Units that receive signals from $B$ cones lie inside the unit diagonals; those for which $W_{b}$ is less than -0.1 are represented by filled circles.

In each panel of Figure 12, many points lie in the upper-left and lower-right quadrants, and mark cells driven by opposed inputs from $\mathrm{R}$ and $\mathrm{G}$ cones. For simple cells $(B)$ and complex cells $(C)$, substantially greater weight is attached to signals from $\mathrm{R}$ cones than to signals from $\mathrm{G}$ cones; for linear nonoriented cells $(A)$ signals from the 2 classes of cones are more equally weighted, as they are for neurons in parvocellular l.g.n. $(D)$. In the population of simple cells (Fig. $12 B$ ), 17 of 48 neurons were driven by $R$ and $G$ cone inputs of the same polarity; 11 of 56 complex cells were driven by inputs of the same polarity.

The results in Figure 12, which show that most neurons in striate cortex have chromatically opponent receptive fields, appear to be at odds with the results in Figure $11, B$ and $C$, which shows that most simple and complex cells prefer nearly achromatic stimuli. The apparent contradiction arises because onc normally expects cells that have opposed inputs from different classes of cones to show overt chromatic opponency of the kind revealed by a biphasic spectral sensitivity curve. In fact, consideration of the spectral scnsitivitics of $R$ and $G$ cones shows that a neuron will be overtly opponent only if the opposed inputs from these cones are well-balanced. A modest preponderance of input from either class of cone, but particularly the $\mathrm{R}$ cone, will leave a cell responding well to achromatic stimuli. For most simple and complex cells inputs from $\mathrm{R}$ cones were strongly preponderant: Figure 13, $A$ and $B$, shows the distribution of the logarithm of the ratio of weights $W_{r} / W_{g}$ for the populations of simple and complex cells represented by Figure $12, B$ and $C$. For both simple and complex cells the modal value of the ratio is 2.5 .

\section{Reliability of measurement}

The parameters $\theta_{m}$ and $\phi_{m}$ provide a worthwhile chromatic signature only if they are reliably measured. Descriptive and inferential statistical methods exist for the treatment of spherical measures (Mardia, 1972), and we have used these to computc the $95 \%$ confidence limits for the mean directions $\left(\theta_{m}\right.$ and $\left.\phi_{m}\right)$ for sets of measurements obtained from 6 haphazardly selected cells. For each cell, we calculated $\theta_{m}$ and $\phi_{m}$ separately for each of the 20 presentations of the full set of stimuli modulated in different directions. The variance of the sample of measures was calculated using procedures given by Mardia (1972, pp. 218-222). The distance from the sample mean direction to the confidence limit was $2.4,3.3,7.5,9.1,10.0$, and 16.7 degrees 

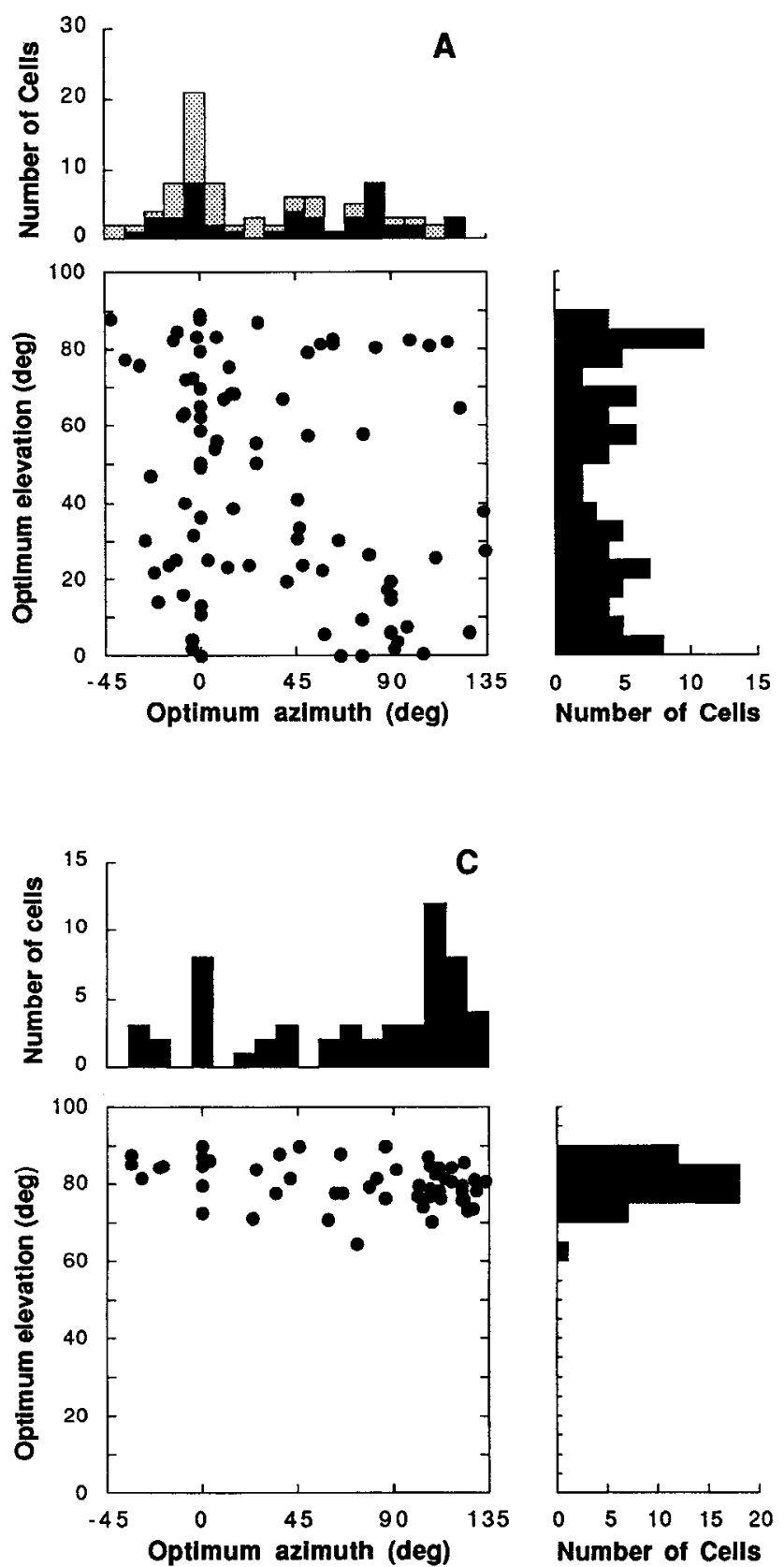
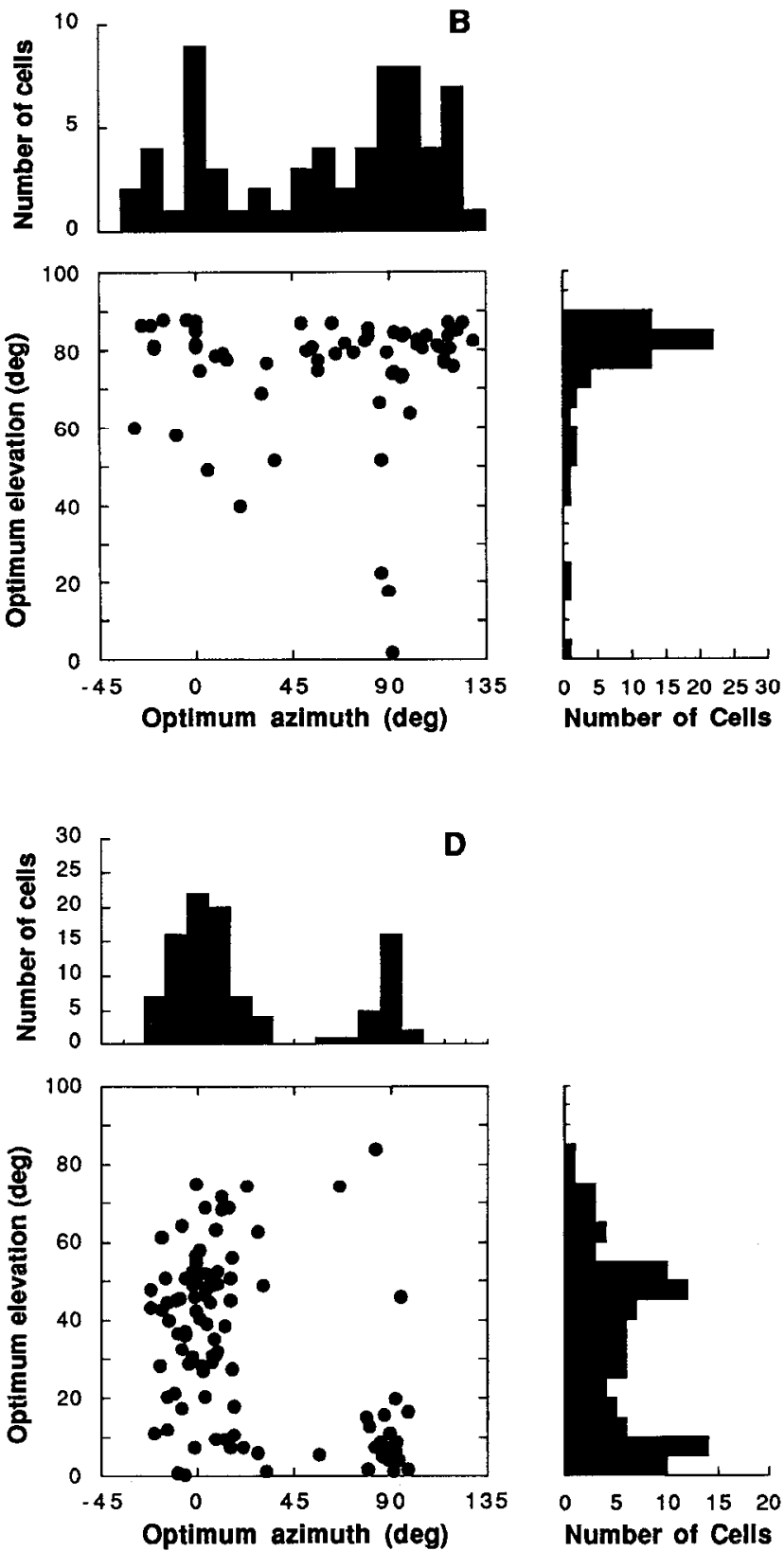

Figure 11. Distribution of azimuth $\left(\theta_{m}\right)$ and elevation $\left(\phi_{m}\right)$ of maximal response. $A$, Nonoriented cells (48). The histogram above the scatter plot shows the distribution of $\phi_{m}$; that to the right, the distribution of $\theta_{m}$. In the histogram of $\phi_{m}$, solid components denote cells with $\theta_{m}<45^{\circ}$; shaded components denote cells with $\theta_{m}>45^{\circ}$. B, Simple cells (48); other details as for $A$. $C$, Complex cells (56); other details as for $A$. $D$, Neurons in parvocellular l.g.n. (from Derrington et al., 1984); other details as for $A$.

for the cases tested (Mardia, pp. 260-261). That is, the probability is 0.95 that the true means lay within these distances from the sample means.

If $\theta_{m}$ is near $90^{\circ}$, a relatively small confidence interval expressed in angular terms can disguise larger uncertainty in the weights that the neuron attaches to inputs from the different classes of cones. This reflects the fact that $\theta_{m}$ for both $\mathrm{R}$ and $\mathrm{G}$ cones lies close to $90^{\circ}$.

\section{Sensitivity to chromatic modulation}

$\theta_{m}$ and $\phi_{m}$, or the relative cone weights, provide a chromatic signature but say nothing about the absolute sensitivity of a neuron. The scale factor $K$ (Eq. 1) provides a measure of sensitivity to modulation, but $K$ depends upon the units in which contrast is measured. One widely used definition represents the contrast of a heterochromatic grating as the Michelson contrast of its 2 out-of-phase homochromatic components, but this measure is physiologically unrealistic because it takes no account of the spectral composition of the components. Even for an optimally chosen pair of lights, the maximum attainable modulation of the quantum catches in $\mathrm{R}$ or $\mathrm{G}$ cones is about $25 \%$; mostly it is much less. Lennie and D'Zmura (1988) discuss other measures of contrast, but all have attendant disadvantages. We need not be concerned about units if we ask only how the sen- 

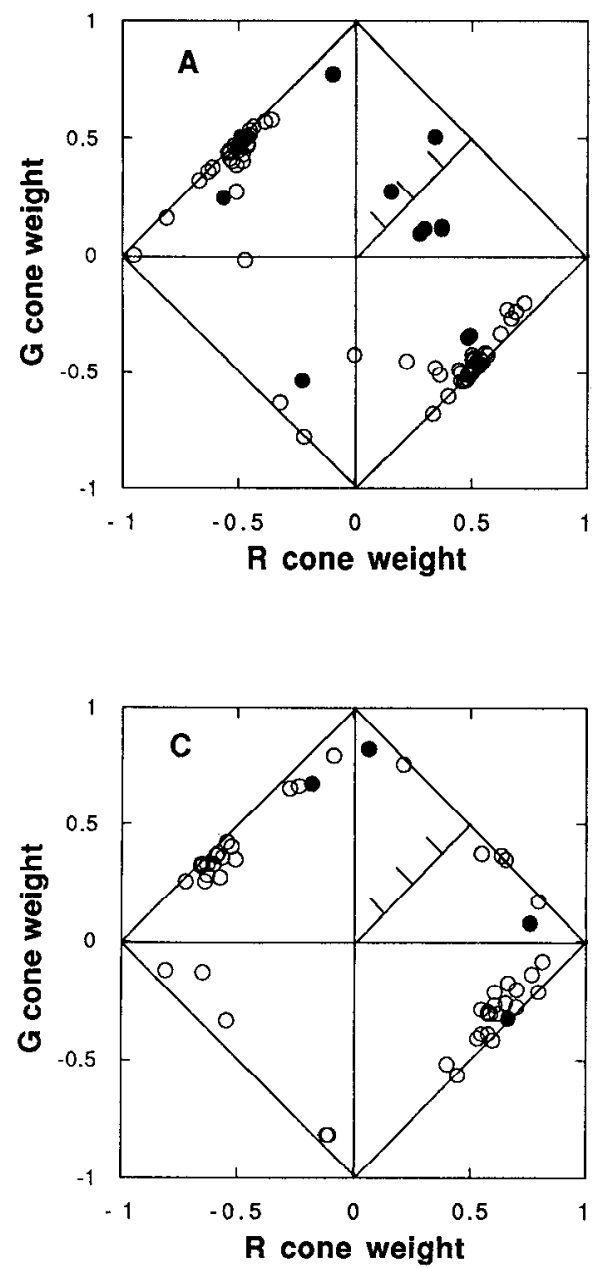
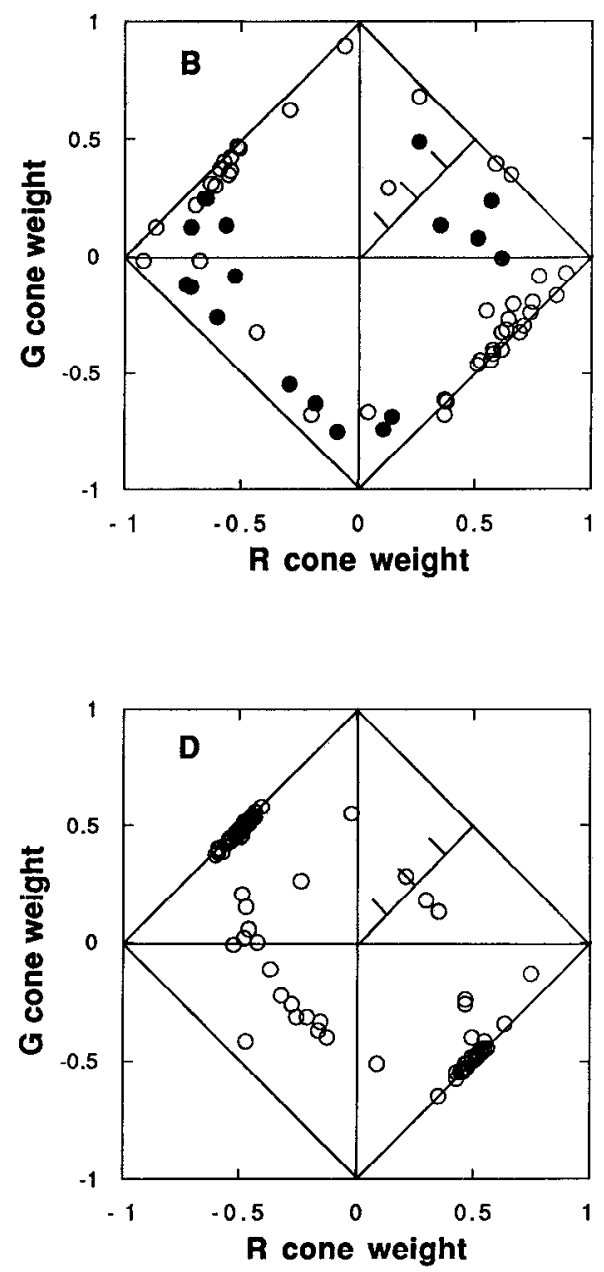

Figure 12. Scatter diagrams showing the weights attached to the modulation of quantum catch in each class of cone. $A$, Nonoriented cells (48). Weights are normalized so that the sum of the unsigned weights $W_{r}+W_{g}+W_{b}$ is $1 . W_{g}$ is plotted against $W_{r}$. Cells that receive input only from $R$ and $G$ cones are represented by points that lie on the unit diagonals; $W_{b}$ is proportional to the perpendicular distance of a point from its nearest diagonal, as shown by the scale in the upper-right quadrant. Cells for which $W_{b}$ is negative are marked by filled points. $B$, Simple cells (48); other details as for $A$. C, Complex cells (56); other details as for $A . D$, Neurons in parvocellular l.g.n. (from Derrington et al., 1984); other details as for $A$. sitivities of individual neurons are related to the sensitivity of the psychophysical observer and use the same units for both physiological and psychophysical observations.

Psychophysical measures of sensitivity are derived from judgments of the minimum modulation that can be reliably detected. The task is usually represented as the problem of detecting a signal in noise. A comparable physiological threshold can be calculated by finding what stimulus evokes a reliable response from a cell. What constitutes a reliable response depends on the individual neuron: for cells that have no maintained discharge (mostly simple cells), any response to stimulus modulation will be reliable, and a stimulus that evokes a single impulse can be detected; for cells that have a maintained discharge (often complex cells), we have to take account of the noisiness of the discharge when setting a criterion for a reliable response. One useful criterion is a discharge that would arise on only $2 \%$ of occasions in the absence of a stimulus, and we have used this in the following illustrations. For the average complex cell, the criterion response was a rise in the average discharge rate of $11 \mathrm{imp} /$ sec; for nonoriented cells, a rise in the modulated response of $10 \mathrm{imp} / \mathrm{scc}$. For the most sensitive nonoriented cells that preferred modulation along the constant $B$ axis, the mean threshold was 3-4 times that of a human observer viewing a chromatically modulated patch of approximately the same size as the receptive field; for cells that preferred modulation along the constant R,G axis, the mean threshold was almost the same as that of the human observer.

\section{Interdependence of spatial and chromatic properties of} receptive fields

A comprehensive description of the receptive field (including both spatial and chromatic properties) could be obtained by measuring responses to modulation along different vectors in the color space for a range of spatial frequencies and orientations. The large number of measurements required made it impracticable to do this; instead, we examined 2 aspects of spatiochromatic interaction: how a large change in spatial frequency affects the chromatic signature of cells and how a large change in the direction of the vector of chromatic modulation affects the spatial tuning of cells.

\section{Variation of chromatic properties with spatial frequency}

If the chromatically opponent mechanisms of a neuron have different spatial tuning characteristics, the chromatic signature of a cell will depend upon the spatial frequency used in the measurement. The center-surround organization of receptive fields in the l.g.n. results in the chromatic signature of a neuron varying substantially with the spatial frequency of the stimuli used to study it, and indeed, the analysis made by Derrington et al. (1984) suggested that this results in confounding of chromatic and achromatic signals. Psychophysical considerations, and also an examination of ways in which signals from l.g.n. might be analyzed to yield distinct chromatic and achromatic mechanisms (D'Zmura and Lennie, 1986), suggest that a purely 

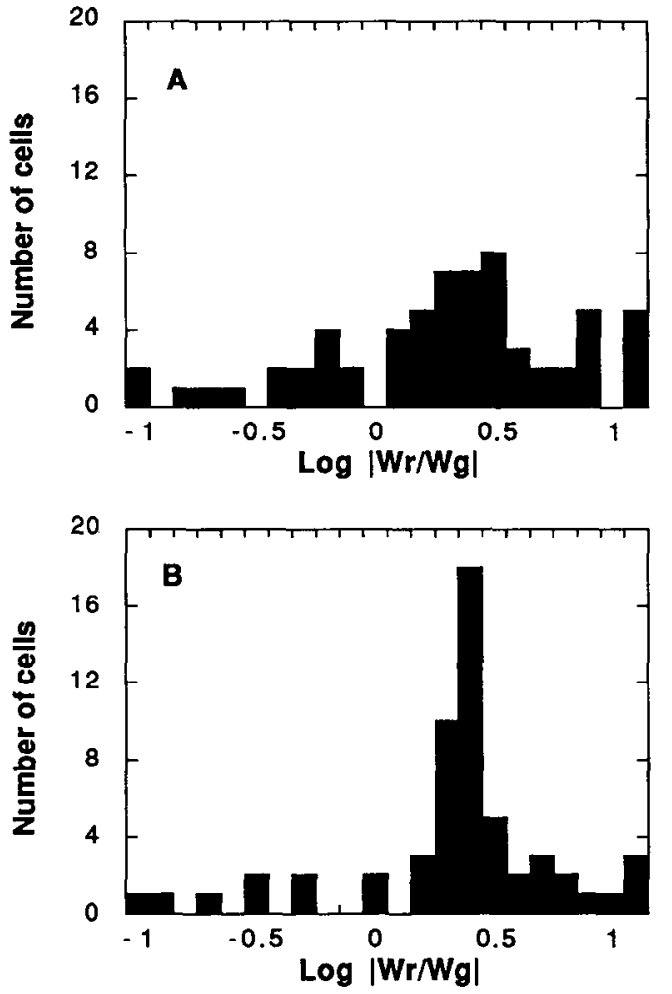

Figure 13. Distribution of the ratio of unsigned weights attached to the modulation of the quantum catches in $\mathrm{R}$ and $\mathrm{G}$ cones for simple $(A)$ and complex $(B)$ cells. Histograms show the logarithm of the ratio $\left|W_{r} / W_{g}\right|$. The median R:G ratio of sensitivities is 1.6 (mean, 3.8) for both types of cell.

achromatic mechanism will be rclatively insensitive to low spatial frequencies, while a purely chromatic one will be most sensitive to low spatial frequencies, and that the chromatic properties of both mechanisms will be stable to variations in the spatial characteristics of the stimulus.

Since most simple and complex cells respond to relatively narrow ranges of spatial frequency, their chromatic properties must be relatively unaffected by changes in the spatial properties of stimuli. Some simple cells and many linear nonoriented cells responded well to both moving gratings and temporally modulated uniform fields. Figure 14, $A$ and $B$, shows, for nonoriented and simple cells whose chromatic properties were examined both with spatially uniform fields and with gratings, how $\theta_{m}$ was altered by the change of spatial frequency. For both groups of cells (though more so for the nonoriented), $\theta_{m}$ was generally lower when measured with a spatially uniform field. This behavior resembles that of neurons in parvocellular l.g.n. (cf. Derrington et al., 1984, Fig. 8) and implies that one of the chromatically opponent mechanisms in the receptive field resolved higher spatial frequencies than the other. However, it should be borne in mind that at middle spatial frequencies, uncorrected chromatic aberration may manifest itself as chromatic opponency in neurons that are not at all chromatically opponent and may lead to overestimates of the spatial opponency in neurons that have nearly coextensive chromatically opponent mechanisms (Flitcroft, 1989). We return to this question in the Discussion. The relation between cone weights and $\theta_{m}$ is nonlinear (Eq. 4). Thus, in interpreting Figure 14 it should also be borne in mind that $\theta_{m}$, if small, will be substantially altered by a small change in the relative weights that a neuron attaches to signals from the different classes of cone.
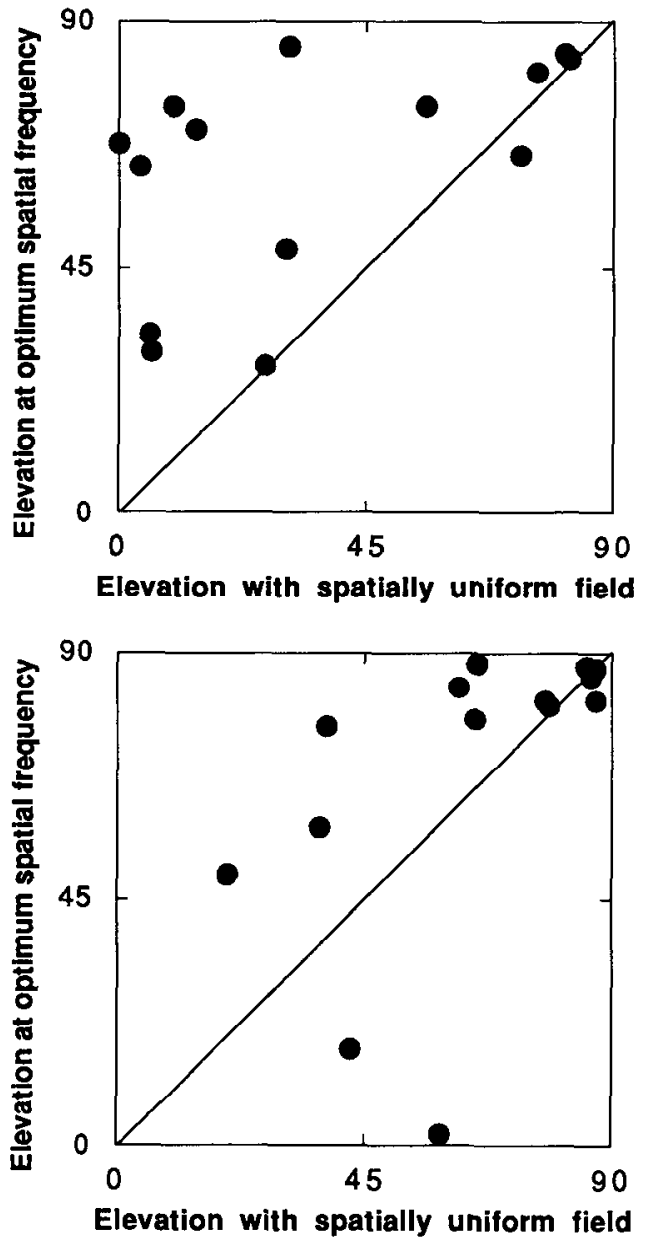

Figure 14. Effect of spatial frequency on the chromatic properties of nonoriented cells $(A)$ and simple cells $(B)$. For those neurons whose chromatic properties could be characterized using both gratings and spatially uniform fields, $\theta_{m}$ estimated from responses to gratings of the optimal spatial frequency is plotted against $\theta_{m}$ estimated from responses to a spatially uniform field modulated along the same vectors. $\theta_{m}$ is generally lower when measured with uniform fields.

\section{Variation of spatial properties with chromaticity of modulation}

If the chromatically opponent mechanisms in a receptive field have different spatial properties, the spatial selectivity of the neuron should depend upon the chromatic composition of the stimulus. This is well-established for neurons in parvocellular l.g.n., where the center-surround arrangement of most receptive fields results in the spatial selectivity being band-pass for achromatic stimuli and low-pass for heterochromatic ones (DeValois et al., 1977; Derrington et al., 1984).

For those cortical neurons that could be driven by both achromatically modulated stimuli and chromatically modulated ones, we measured the spatial selectivity with an achromatic stimulus and with a chromatic one modulated along the vector $\theta=0, \phi=\phi_{m}$.

\section{Linear nonoriented cells}

Figures $2 A$ and $4 A$ show the spatial selectivities of 2 nonoriented cells whose other properties were described earlier. Figure 15 shows the selectivities of 4 other cells. 

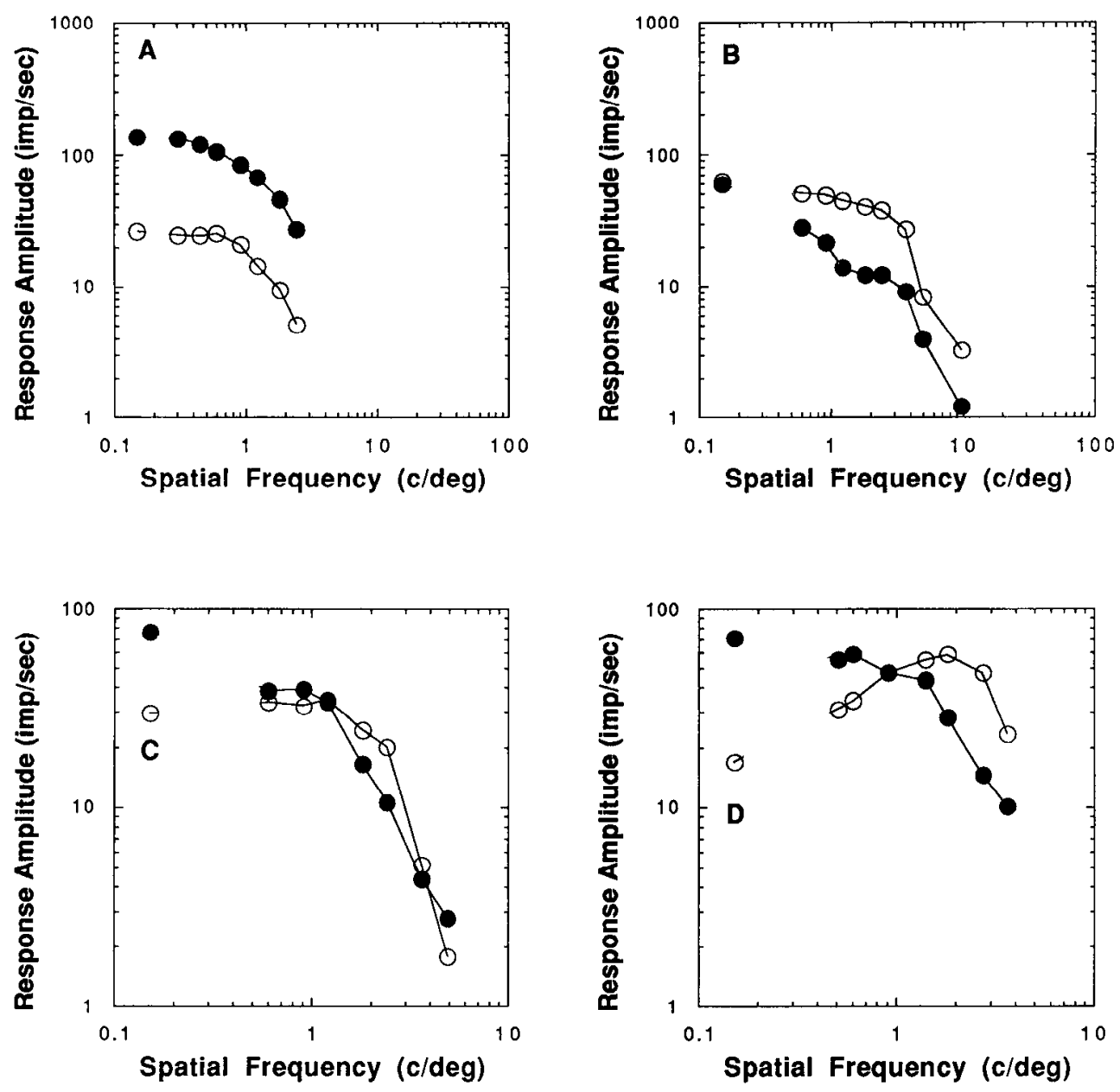

Figure 15. Spatial frequency characteristics of nonoriented cells, measured with gratings modulated along the achromatic axis (open circles) and the direction in the isoluminant plane corresponding to $\phi_{m}$ (filled circles). $A$, Layer VI cell; $B$, cell in unknown layer; $C$, layer VI neuron; $D$, layer VI neuron. This was 1 of only 2 nonoriented neurons that was less responsive to a grating than to modulation of a spatially uniform field.

The spatiochromatic properties of nonoriented cells are of some interest in view of reports (Michael, 1978a-c, 1985; Livingstone and Hubel, 1984) that many neurons in striate cortex have concentrically organized "double-opponent" receptive fields, with center and surround each containing chromatically opponent mechanisms of opposite sense (e.g., $\mathrm{R}+\mathrm{G}-$ center, $\mathrm{R}-\mathrm{G}+$ surround). A cell with a receptive field like this would be selective for the size of a stimulus containing chromatic contrast and should respond well to a heterochromatic grating of the appropriate spatial frequency, less well to chromatic modulation of a spatially uniform field, and not at all to achromatic stimuli. Of the 29 nonoriented cells on which we obtained measurements of spatial selectivity for both chromatic and achromatic gratings, only 2 were less sensitive to chromatic modulation of a spatially uniform field than to a moving grating. These cells responded well to achromatic gratings. Moreover, in all but one case in which we found some effect of spatial frequency on the chromatic signature of a cell, the cell's preference always shifted towards achromatically modulated stimuli as spatial frequency was raised (Fig. 14A). Our observations suggest that double-opponent cells are rare.

\section{Simple cells}

Figures $5 A$ and $6 A$ show the spatial selectivities of simple cells whose other properties were characterized earlier. Figure 16 shows 4 further examples. Open circles indicate responses to achromatic gratings, filled circles responses to chromatically modulated gratings. Although measurements were attempted on most simple cells, we obtained satisfactory measurements of the spatial selectivity for both chromatic and achromatic gratings from only 20 , since most did not respond reliably to chromatic gratings. Of the simple cells that responded to both types of gratings, several were most sensitive to chromatic modulation at low spatial frequencies and to achromatic modulation at higher spatial frequencies (e.g., Fig. 16, $A, B$ ). This greater selectivity for achromatic than chromatic patterns is consistent with Figure $14 B$ and implies that the spatially antagonistic regions of the receptive field have different spectral sensitivities. Six simple cclls ( 3 of which wcrc known to lie in layer II/III) were more sensitive to some chromatic grating than to chromatic modulation of a spatially uniform field. Figure $16, C$ and $D$, shows the curves obtained from 2 of these.

\section{Complex cells}

For the 21 complex cells on which we were able to make measurements, the loss of responsiveness to chromatic gratings as spatial frequency dropped below its optimum was always pronounced. Results from 2 neurons are shown in Figures $7 A$ and $9 A$, and Figure 17 shows the spatial selectivities of 4 more. Hubel and Wiesel (1968), Michael (1978b, 1985), and Thorell et al. (1984) have described cells with similar spatial selectivity for chromatic stimuli. With rare exceptions, the spatial properties of complex cells were independent of the chromaticity of the stimuli used to measure them. Our observations on this 

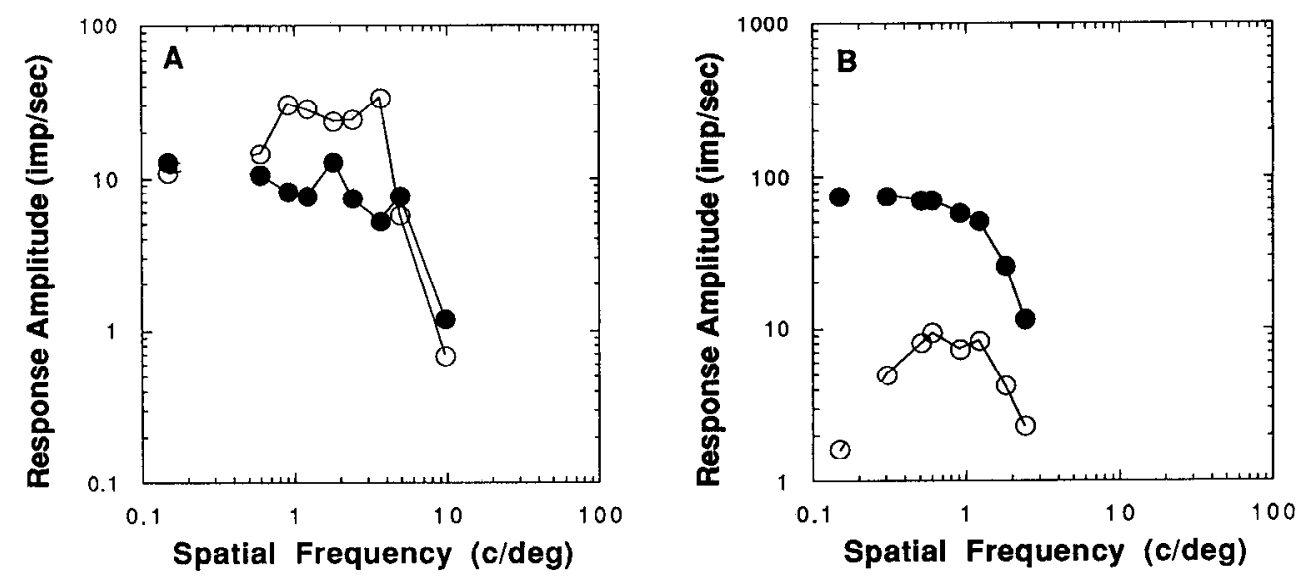

Figure 16. Spatial frequency characteristics of simple cells. Selectivity for spatial frequency was measured with gratings modulated along the achromatic axis (open circles) and the direction in the isoluminant plane corresponding to $\phi_{m}$ (filled circles). $A$, Cell in unknown layer; $B$, cell in unknown layer; $C$, cell in layer II/III, outside a region of dense cytochrome oxidase; $D$, neuron in unknown layer.
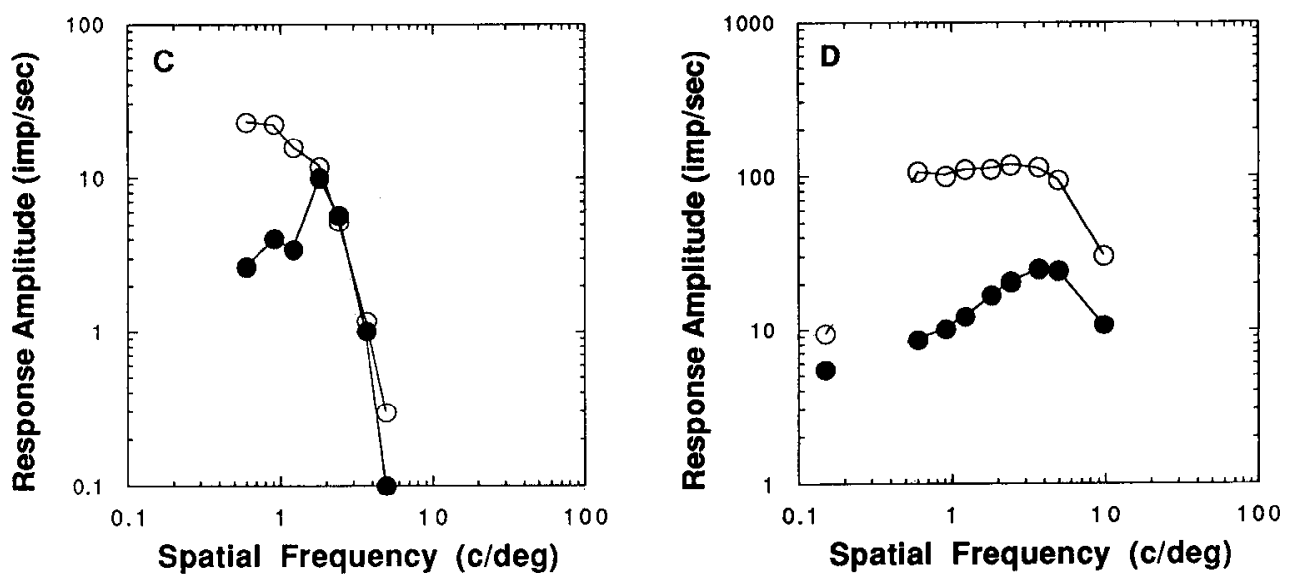

point agree with those of Thorell et al. (1984). The properties of these complex cells are most simply explained by supposing that the receptive field is comprised of linearly summing subunits of the kind postulated by Spitzer and Hochstein (1985b) and that these subunits are spatially selective, with the same chromatic sensitivities in their opponent parts.

\section{Distribution of neurons in cortex}

For a variety of reasons (such as dimpling of cortex or failure unambiguously to pinpoint a lesion), laminar positions are known certainly for only 198 cells ( 1 in layer I, 75 in layer II/III, 21 in layer IVA, 16 in layer IVB, 17 in layer IVC $\alpha, 19$ in layer IVC $\beta$, 12 in layer V, and 37 in layer VI).

Sensitivity to chromatic modulation is apparently closely allied with sensitivity to low spatial frequencies. Layers IVC $\beta$ and IVA contained a relatively large concentration of neurons that responded well to modulation of spatially uniform fields ( 21 of 39). Of these, 10 had $\theta_{m}$ of less than $45^{\circ}$. Neurons that responded well to spatially uniform fields were also relatively common in layer VI (26 of 40), and of these 10 had $\theta_{m}$ less than $45^{\circ}$. In other layers, very few cells responded to modulation of uniform fields.

Outside layer IV, striate cortex contains regularly spaced regions ("blobs") that in histological sections stain densely for the mitochondrial enzyme cytochrome oxidase (Horton and Hubel, 1981; Humphrey and Hendrickson, 1983; Livingstone and Hubel, 1984). Where possible, we distinguished cells lying within and between blobs, but in several animals the cytochrome reaction in the region of cortex underlying the craniotomy was substantially weaker than in adjacent cortex, and in these animals it was not possible unequivocally to assign cells to blobs or to the interstices.

Neurons in layer II/III are of special interest in view of reports (Livingstone and Hubel, 1984; Ts'o and Gilbert, 1988) that blobs are particularly rich in chromatically opponent neurons. Figure 18 shows the distribution of $\theta_{m}$ and $\phi_{m}$ for all the neurons known to be in layer II/III. If the measurement was made at more than one spatial frequency, the parameters were estimated from the one to which the cell was most sensitive. In no case did a neuron respond most strongly to modulation of a spatially uniform field. Neurons positively identified as lying in blobs are marked by filled circles, and those known to be in the interstices are marked by open circles. Of the other neurons, those that were not orientation-selective (and therefore more likely to lie in blobs) are marked by filled squares, and those that were orientation-selective are marked by open squares. In this sample, there is evidently no clustering of chromatic properties that can be easily tied to a cell's presence in a region rich in cytochrome oxidase. Six of the neurons encountered had $\theta_{m}$ less than $45^{\circ}$, but there was no indication of a population of cells specially sensitive to chromatically modulated stimuli. Indeed, the most distinctive characteristic of the population of neurons in layer II/III was the paucity of cells that showed strong preferences for chromatically modulated stimuli. We consider in the Discussion how our findings are related to earlier work. 

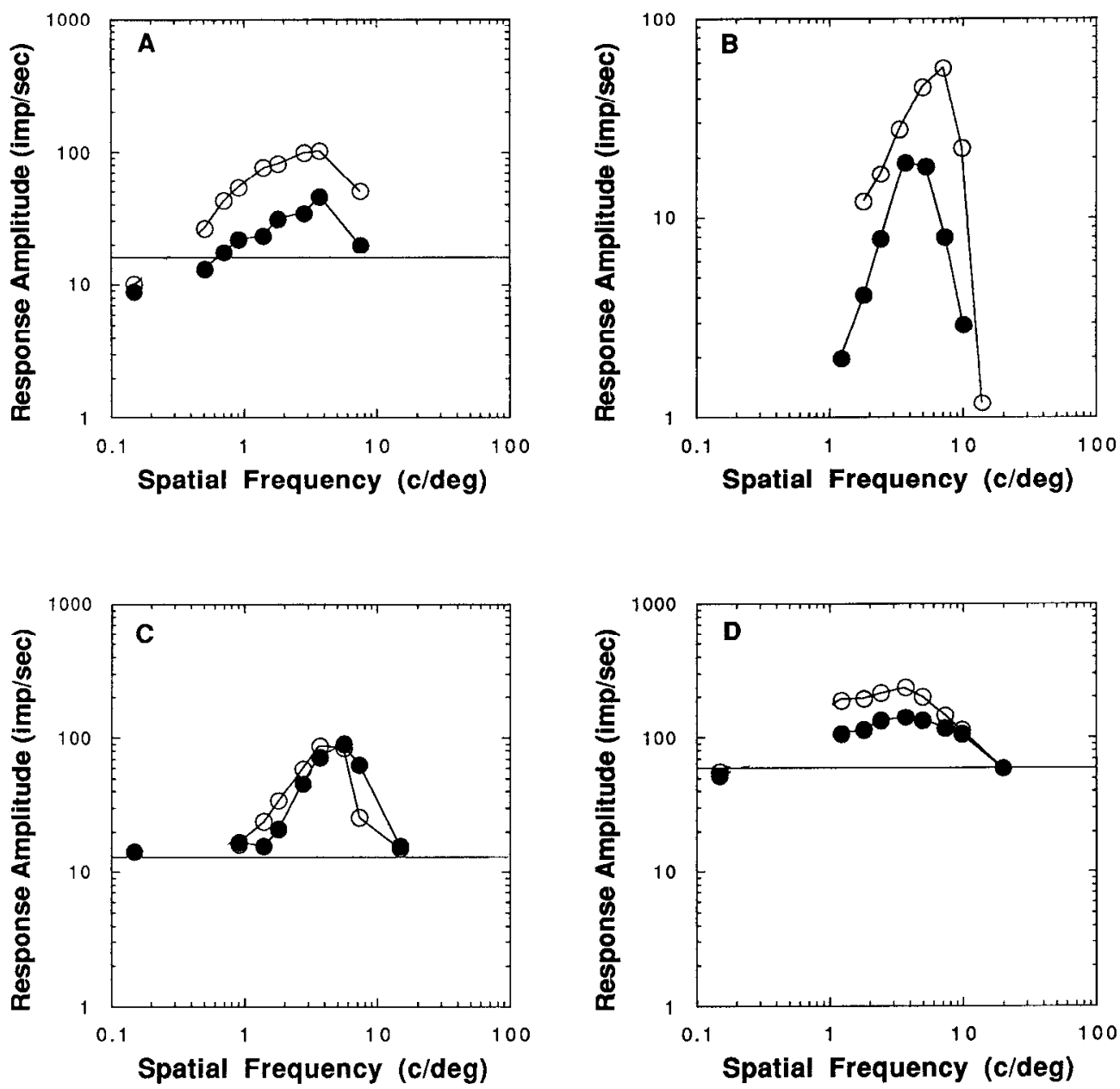

Figure 17. Spatial frequency characteristics of complex cells, measured with gratings modulated along the achromatic axis (open circles) and the direction in the isoluminant plane corresponding to $\phi_{m}$ (filled circles). The horizontal line on each plot marks the maintained discharge to the adaptation point.

\section{Discussion}

Transformation of signals from I.g.n.

With rare exceptions, the behavior of neurons in all layers of striate cortex is described well by a model in which a cell responds to the weighted sum of signals from the 3 classes of cones (Eq. 1) or to the rectified sum of these signals (Eq. 2). For a particular spatial configuration, our model provides a succinct and complete description of the chromatic properties of neurons and can be used to predict the response of a neuron to stimuli of any chromaticity and luminance. Moreover, we can deduce the weights with which the neuron combines signals from the 3 classes of cone. The completeness of description and the sensitivity of measurements on cells in a neutral state of adaptation are the principal advantages of our technique over others (e.g., the measurement of spectral sensitivity) that are often used to characterize the chromatic properties of neurons.

\section{Chromatic selectivity}

The fact that Eq. 1 satisfactorily describes the behavior of neurons in 1.g.n. and linear (simple and nonoriented) neurons in striate cortex means that the cortical neurons are no more sharply tuned for chromaticity than are neurons in the l.g.n. Moreover, since the behavior of most complex cells is well described by Eq. 2, these too must have the same selectivity as linear cortical neurons and neurons in l.g.n. On this point our observations agree with those of DeMonasterio and Schein (1982) but differ from those of Thorell et al. (1984), who found simple cells to be more selective. Thorell et al. may have encountered (and treated as simple cells) narrowly tuned cells that we distinguished from simple cells. We found 6 neurons that showed sharp selectivity for chromatic modulation along vectors on, or close to, the constant B axis; we know too little to develop an adequate account of their behavior. We could have overlooked some neurons that had even sharper selectivity, since in most experiments responses to chromatic modulation were sampled at $45^{\circ}$ intervals.

\section{Chromatic preferences}

Although the chromatic selectivities of most cortical neurons are the same as those of neurons in l.g.n., cortical neurons generally have distinctively different chromatic preferences. This is evident from the distributions of $\theta_{m}$ and $\phi_{m}$ (Fig. 11) and from the distributions of cone weights (Fig. 12).

Although almost all neurons in striate cortex show some degree of chromatic opponency, only in the population of linear nonoriented cells was this pronounced, and even here the distribution of $\phi_{m}$ (the azimuth of preferred modulation) showed little of the crisp bimodality seen for parvocellular l.g.n. (Fig. 11 ). Some neurons clearly preferred modulation along vectors that lay neither along the constant $B$ axis nor along the constant $\mathrm{K}, \mathrm{G}$ axis. Thorell et al. (1984) also found a broad but bimodal distribution of chromatic preferences in their population of sim- 


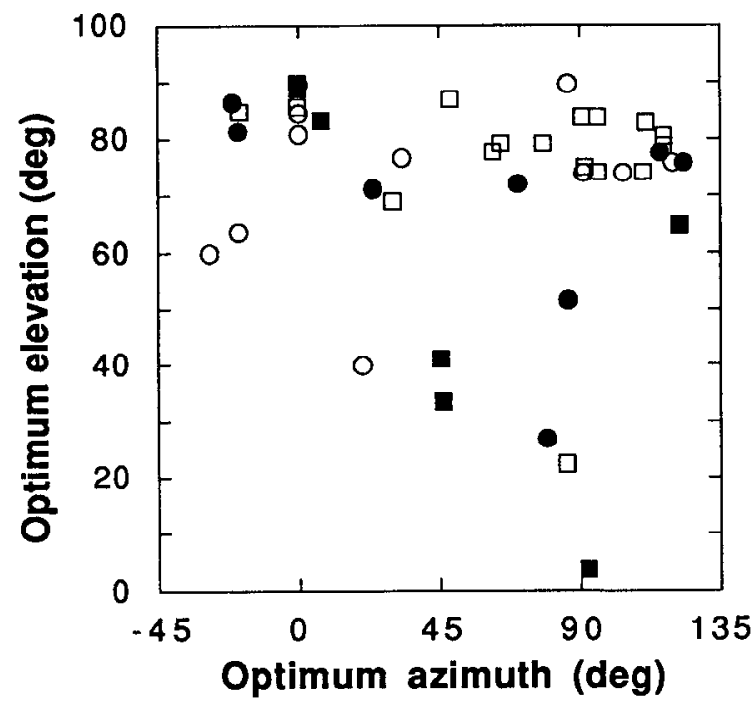

Figure 18. Distribution of $\theta_{m}$ and $\phi_{m}$ for all neurons known to be in layer II/III. These parameters were estimated using stimuli of the optimal spatial configuration - for some cells, a spatially uniform field; for most, a grating. Filled circles, units lying within regions rich in cytochrome oxidase; open circles, neurons known to lie outside regions rich in cytochrome oxidase; filled squares, units whose positions in relation to cytochrome oxidase blobs are unknown but which were orientation selective; open squares, units whose positions in relation to cytochrome oxidase blobs were unknown but which lacked orientation selectivity.

ple cells, which included linear nonoriented cells of the kind encountered here.

The weak chromatic opponency shown by most simple and complex cells distinguished them from nonoriented cells and from neurons in 1.g.n. Moreover, the chromatic properties of most simple and complex cells were stable to changes in spatial frequency. The fact that virtually all neurons in striate cortex are chromatically opponent revives the question (raised earlier by our work on l.g.n.) of whether chromatic opponency per se is of any functional significance. The chromatic opponency found in almost all neurons in 1.g.n. is not taken to imply that their exclusive or principal role is to convey chromatic signals. Similarly, the prevalence of chromatic opponency in cortex emphasizes that mere possession of the property is poor evidence for a role in color vision, and the weak opponency found in most simple and complex cells may have little significance. To evaluate a cell's probable contribution to color vision, it is obviously necessary to characterize its chromatic properties fully and to consider these in relation to its other visual attributes.

\section{Construction of receptive fields}

The analysis of the weights with which cortical cells combine signals from different classes of cone (Figs. 12,13) suggests that they receive simple combinations of signals from cones. Within a single R-G neuron in l.g.n. (the class that must provide the dominant input to cortical cells), the signals from $R$ and $G$ cones are well balanced, yet simple and complex cells receive a preponderant input from $\mathrm{R}$ cones. The question therefore arises as to how this imbalance is produced in cortex.

D'Zmura and Lennie (1986) described how canonical "chromatic" and "achromatic" mechanisms could be created simply by the addition of signals from 2 neurons in l.g.n. that had chromatically opponent receptive fields in the same place. Sim- ple combination rules also can give rise to cells that are driven predominantly by one class of cone. We need to assume that $\mathrm{R}$ and $G$ cones do not occur in equal numbers and that the imbalance of weights in a cortical cell reflects an imbalance in the numbers of ganglion cells and 1.g.n. cells with centers driven by the 2 types of cones. There is good reason to believe that in and close to the fovea each cone drives 2 midget ganglion cells (one plausibly on-center, the other off-center) and that the center of the receptive field of each ganglion cell is driven by a single cone (Schein, 1988). Suppose further that $\mathrm{R}$ cones are twice as numerous as $\mathrm{G}$ cones, an idea for which there is considerable psychophysical evidence (Cicerone and Nerger, 1989; Vimal et al., 1989). In the fovea there will be twice as many midget ganglion cells with receptive field centers driven by $\mathrm{R}$ cones as there are cells with centers driven by $\mathrm{G}$ cones. We presume (from the known close match between the number of optic nerve fibers and the number of relay cells in the l.g.n.) that each midget ganglion cell drives a single neuron in parvocellular l.g.n. This scheme forbids overlap of receptive field centers of ganglion cells or parvocellular neurons (except for an on/off center pair), so cortical cells that combine signals from parvocellular neurons must have receptive fields larger than those of the cells that drive them. Addition of signals from l.g.n. neurons whose receptive field centers lie along a line will produce a receptive field that has an elongated strip of high sensitivity driven by $R$ and $G$ cones in whatever proportions they occur along that line; a $2: 1$ ratio of $\mathrm{R}$ to $\mathrm{G}$ cones will give rise to correspondingly weighted signals.

\section{Consequences of chromatic aberration}

The focal length of the eye varies monotonically with wavelength, with the result that if the eye is in focus for one wavelength, it will be out of focus for all others. The differential defocus brought about by this longitudinal chromatic aberration may be as large as 3 diopters and will, for example, introduce achromatic contrast in a nominally isoluminant heterochromatic grating. We therefore need to understand the effect of chromatic aberration in interpreting the responses to heterochromatic gratings.

Flitcroft (1989) has examined the effects of chromatic aberration on model neurons with receptive fields that have chromatically opponent mechanisms that are spatially coextensive and has shown that for a model eye and grating patterns of the kind used in our experiments, the effects of chromatic aberration are negligible if neurons receive inputs from only $R$ and $G$ cones. For neurons that receive inputs from $B$ cones opposed to some combined input from $\mathrm{R}$ and $\mathrm{G}$ cones, Flitcroft's analysis shows that as a result of chromatic aberration $\theta_{m}$ will rise as spatial frequency is raised, even though the receptive field is not spatially opponent. This effect may have contributed to the spatialfrequency dependent change in $\theta_{m}$ for 1 or 2 of the nonoriented cells shown in Figure 14A, but for the simple cells characterized in Figure $14 B$, it is securely ruled out by the band-pass form of the spatial-frequency characteristic measured with achromatic gratings.

\section{Laminar distribution of sensitivity to chromatic modulation}

The neurons most sensitive to chromatic modulation lay mainly in layers IVA, IVC $\beta$, and VI and were least frequently seen in layers II/III and layer V. Other workers have also found chromatically opponent neurons to be relatively common in the 
subdivisions of layer IV, particularly layers IVA and IVC $\beta$ : Gouras (1974) found them more often in layer IVC $\beta$ (his layer $4 B$ ) than elsewhere; Livingstone and Hubel (1984) found that more than half the neurons studied in layer IVC $\beta$ were color opponent; Dow (1974) and Dow and Gouras (1973) found coloropponent units most often in the region of layer IVA (their 3B); Vautin and Dow (1985) found "primary color" cells most often in layers IVC $\alpha$ and IVA. Michael (1985) concluded that most cells in layers IVA and IVC $\alpha$ were "color coded," with doubleopponent receptive ficlds. We saw no such cclls in layer IV; we have no explanation for the disagreement. Our most surprising finding was the relatively high frequency with which we encountered strongly opponent neurons in layer VI. No other physiological study has reported either a concentration of chromatically sensitive neurons or a concentration of neurons that lacked orientation selectivity, but in anatomical studies Tootell et al. (1988) have drawn attention to the fact that in layers V and VI there is strong patchy uptake of $\left[{ }^{14} \mathrm{C}\right] 2$-deoxy- $d$-glucose when the macaque is exposed to a spatially uniform field flickering between red and an isoluminant gray.

\section{Cells in layer $I I / I I I$}

Livingstone and Hubel (1984), and Ts'o and Gilbert (1988) found in layer II/III an abundance of chromatically opponent units in the blobs and a dearth of them in the interstices. Both groups reported that in the blobs about $70 \%$ of neurons with "nonoriented" receptive fields were chromatically opponent. Our results are consistent with the observation that neurons in blobs are chromatically opponent, but they challenge the observation that chromatically opponent units are largely confined to the blobs: nearly all neurons encountered in our experiments gave discernible responses to isoluminant chromatic modulation, and these responses would have been a little stronger had we used saturated lights of the kind used in the other studies. By a $U$ test, there is no significant difference (at the $5 \%$ level) between the preferred elevations of the cells in blobs and outside them. It is not clear that different workers use the same criteria to demarcate blobs. Moreover, we studied only 74 neurons in layer II/III, and as a consequence we might not have encountered the full range of properties observed in the other studies. Nevertheless, we might have expected to see signs of the major groups identified by others.

One important difference between our experiments and the earlier ones is that our measurements provide a comprehensive description of the chromatic properties of neurons. In contrast, a simple division of cells into chromatically opponent and nonopponent classes provides little information about the kinds of stimuli to which the cells are most responsive and may lead one to divide the population where no objective boundary exists. Classification of cells into "opponent" and "nonopponent" groups may also obscure important differences in sensitivity to chromatically modulated stimuli. For example, although our units with strong chromatic opponency tended to have nonoriented receptive fields, few of the nonoriented neurons in layer II/III had values of $\theta_{m}$ as low as those commonly found in nonoriented cells in layers IV and VI (cf. Figs. 18 and $11 \mathrm{~A}$ ).

\section{Double-opponent cells}

Since these were first described in goldfish (Daw, 1968), they have occupied a prominent place in the physiology of color vision because they have been thought to play a role in the perceptual phenomena of simultaneous color contrast and color constancy (Daw, 1984). We did not see linearly summing neurons that had classical double-opponent receptive fields, although some linearly summing units (mostly simple cells-see Fig. 16 and accompanying text) and some complex cells (see Fig. 17) responded better to chromatically modulated gratings than to chromatic modulation of spatially uniform fields. However, such cells showed little preference for chromatically versus achromatically modulated stimuli (in only 2 cases was $\theta_{m}$ less than $45^{\circ}$ ). Similar cells have been described before by Thorell et al. (1984). Complex cells would seem to provide a particularly poor substrate for a chromatic pathway since the nonlinearity of signal summation, which causes an unmodulated response to a moving grating, gives rise to indistinguishable responses to complementary excursions in chromaticity from the adaptation point.

Livingstone and Hubel (1984) identified many neurons in blobs as double-opponent, because chromatically opponent responses to small stimuli were diminished as the stimuli were enlarged. This procedure admits as "double opponent" cells with a variety of receptive field organizations, including those in which the surround simply suppresses any activity evoked by stimulation of the chromatically opponent center-cells recently described by Hubel and Livingstone (1987) and Ts'o and Gilbert (1988). It is not clear what role such cells might have in color vision, since they respond poorly to chromatic modulation of a spatially uniform field - the stimulus to which chromatic mechanisms isolated in psychophysical experiments are most sensitive.

\section{Flow of chromatic information from striate cortex}

One puzzling feature of our observations is that layer II/III, which appcars to provide the most substantial projection to extrastriate cortex, contains few neurons with pronounced chromatic opponency. Of those layers that contained neurons with strong chromatic opponency, only layer VI sends known projections to V2 (Kennedy and Bullier, 1985). Perhaps this projection provides a major conduit for chromatic signals.

\section{Relationship to psychophysical work}

A great deal of work (reviewed by Lennie and D'Zmura, 1988) points to the existence of 3 postreceptoral mechanisms that represent the light-dark, red-green, and yellow-blue dimensions of color experience. Several studies have attempted to measure the spatiotemporal properties of postreceptoral chromatic mechanisms by using isoluminant chromatic gratings that are presumed to excite only the red-green or blue-yellow mechanisms (Van der Horst and Bouman, 1969; Mullen, 1985). This work shows that the putative red-green and yellow-blue mechanisms are most sensitive to very low spatial frequencies and have poor spatial resolution. Bradley et al. (1988) have used the same technique to show that chromatic mechanisms have relatively poor orientation sensitivity. Other work (e.g., Robson, 1966) suggests that the achromatic mechanism has a band-pass spatial sensitivity. We might therefore expect the major part of the chromatic signal to be carried by mechanisms that have lowpass spatial contrast sensitivity functions. These considerations implicate nonoriented cells, which are the most sensitive to chromatic modulation at low spatial frequencies, in the detection of chromatic modulations, and they implicate simple and complex cells in the detection of achromatic patterns. However, 
few cells in striate cortex have the properties expected of the canonical chromatic mechanisms.

Because Derrington et al. (1984) found only 2 classes of parvocellular neuron in 1.g.n. (one of which appeared to transmit information about the yellow-blue chromatic dimension, while the other apparently confounded the red-green and the achromatic signals), we had expected to find that cortex would give rise to recognizable substrates of the chromatic and achromatic mechanisms identified in psychophysical experiments. The properties of many simple and complex cells come close to those expected of an "achromatic" mechanism, and although the very sharp chromatic tuning of a small number of nonoriented cells (all with $\theta_{m}$ close to $0^{\circ}$ and $\phi_{m}$ close to $0^{\circ}$ or $180^{\circ}$ ) strongly implicates them in the analysis of chromatic signals, few others behaved as would the putative "red-green" or "blue-yellow" mechanisms: $\theta_{m}$ rarely lay in the isoluminant plane, and although the modal values of $\phi_{m}$ lay along the constant $\mathrm{B}$ axis and constant R,G axis, as might be expected from the psychophysical work of Krauskopfet al. (1982), for many neurons $\phi_{m}$ lay neither along one of these axes nor along axes corresponding to the unique hues. Moreover, the chromatic properties of most neurons that responded to low spatial frequencies varied with the spatial frequency of the stimulus, though not to the degree seen in our earlier work on l.g.n.

We also examined whether the responses of nonoriented cells could become depressed by prolonged exposure to chromatic modulation but found no clear evidence of habituation of the sort observed in analogous psychophysical experiments (Krauskopf et al., 1982).

Thus, while the putative achromatic mechanism seems to be reasonably well developed in striate cortex, there are only modest signs of the red-green and yellow-blue mechanisms whose existence is so firmly established by psychophysics. For these we apparently must look to regions beyond striate cortex.

\section{References}

Baylor, D. A., B. J. Nunn, and J. L. Schnapf (1987) Spectral sensitivity of cones of the monkey Macaca fascicularis. J. Physiol. (Lond.) 390: $145-160$.

Bradley, A., E. Switkes, and K. K. DeValois (1988) Orientation and spatial frequency selectivity of adaptation to color and luminance gratings. Vision Res. 28: 841-856.

Cicerone, C. M., and J. L. Nerger (1989) The relative numbers of longwavelength-sensitive to middle-wavelength-sensitive cones in the human fovea centralis. Vision Res. 29: 115-128.

Daw, N. W. (1968) Color-coded ganglion cells in the goldfish retina: Extension of their receptive fields by means of new stimuli. J. Physiol. (Lond.) 197: 567-592.

Daw, N. W. (1984) The psychology and physiology of colour vision. Trends Neurosci. 7: 330-335.

DeMonasterio, F. M., and S. J. Schein (1982) Spectral bandwidths of color-opponent cells of geniculocortical pathway of macaque monkey. J. Neurophysiol. 47: 214-224.

Derrington, A. M., J. Krauskopf, and P. Lennie (1984) Chromatic mechanisms in lateral geniculate nucleus of macaque. J. Physiol. (Lond.) 357: 241-265.

DeValois, R. L., D. M. Snodderly, Jr., E. W. Yund, and N. K. Hepler (1977) Responses of macaque lateral geniculate cells to luminance and color figures. Sens. Proc. 1: 244-259.

DeValois, R. L., D. G. Albrecht, and L. G. Thorell (1982a) Spatial frequency selectivity of cells in macaque visual cortex. Vision Res. 22: $545-560$.

DeValois, R. L., E. W. Yund, and N. K. Hepler (1982b) The orientation and direction selectivity of cells in macaque visual cortex. Vision Res. 22: 531-544.

Dow, B. M. (1974) Functional classes of cells and their laminar distribution in monkey visual cortex. J. Neurophysiol. 37: 927-946.
Dow, B. M., and P. Gouras (1973) Color and spatial specificity of single units in rhesus monkey foveal striate cortex. J. Neurophysiol. 36: $79-100$

D'Zmura, M., and P. Lennie (1986) Mechanisms of color constancy. J. Opt. Soc. Am. A 3: 1662-1672.

Flitcroft, D. I. (1989) The interactions between chromatic aberration, defocus and stimulus chromaticity: Implications for visual physiology and colorimetry. Vision Res. 29: 349-360.

Gouras, P. (1974) Opponent-colour cells in different layers of foveal striate cortex. J. Physiol. (Lond.) 238: 583-602.

Horton, J. C., and D. H. Hubel (1981) Regular patchy distribution of cytochrome oxidase staining in primary visual cortex of macaque monkey. Nature 292: 762-764.

Hubel, D. H., and M. S. Livingstone (1987) Segregation of form, color, and stereopsis in primate area 18. J. Neurosci. 7: 3378-3415.

Hubel, D. H., and T. N. Wiesel (1968) Receptive fields and functional architecture of monkey striate cortex. J. Physiol. (Lond.) 195: $215-$ 243.

Humphrey, A. L., and A. E. Hendrickson (1983) Background and stimulus-induced patterns of high metabolic activity in the visual cortex (area 17) of the squirrel and macaque monkey. J. Neurosci. 3: 345-358.

Ingling, C. R., Jr., and E. Martinez-Uriegas (1983) The relationship between spectral sensitivity and spatial sensitivity for the primate r-g X-channel. Vision Res. 23: 1495-1500.

Kennedy, H., and J. Bullier (1985) A double-labeling investigation of the afferent connectivity to cortical areas V1 and V2 of the macaque monkey. J. Neurosci. 5: 2815-2830.

Krauskopf, J., D. R. Williams, and D. W. Heeley (1982) Cardinal directions of color space. Vision Res. 22: 1123-1131.

Lennie, P., and M. D'Zmura (1988) Mechanisms of color vision. CRC Crit. Rev. Neurobiol. 3: 333-400.

Levick, W. R. (1972) Another tungsten microelectrode. Med. Biol. Engin. 10: 510-515.

Livingstone, M. S., and D. H. Hubel (1984) Anatomy and physiology of a color system in primate primary visual cortex. J. Neurosci. 4: 309-356

Mardia, K. V. (1972) Statistics of Directional Data, Academic Press, New York.

Michael, C. R. (1978a) Color vision mechanisms in monkey striate cortex: Dual-opponent cells with concentric receptive fields. J. Neurophysiol. $41: 572-588$.

Michael, C. R. (1978b) Color vision mechanisms in monkey striate cortex: Simple cells with dual opponent-color receptive fields. J. Neurophysiol. 41: 1233-1249.

Michael, C. R. (1978c) Color-sensitive complex cells in monkey striate cortex. J. Neurophysiol. 41: 1250-1266.

Michael, C. R. (1985) I aminar segregation of color cells in the monkey's striate cortex. Vision Res. 25: 415-423.

Mollon, J. D. (1982) Color vision. Annu. Rev. Psychol. 33: 41-85.

Movshon, J. A., I. D. Thompson, and D. J. Tolhurst (1978) Receptive field organization of complex cells in the cat's striate cortex. J. Physiol. (Lond.) 283: 79-99.

Mullen, K. T. (1985) The contrast sensitivity of human colour vision to red-green and blue-yellow chromatic gratings. J. Physiol. (Lond.) 359: $381-400$

Robson, J. G. (1966) Spatial and temporal contrast sensitivity functions of the visual system. J. Opt. Soc. Am. 56: 1141-1142.

Schnapf, J. L., T. W. Kraft, and D. A. Baylor (1987) Spectral sensitivity of human cone photoreceptors. Nature 325: 439-441.

Schein, S. J. (1988) Anatomy of macaque fovea and spatial densities of neurons in foveal representation. J. Comp. Neurol. 269:479-505.

Sclar, G., J. H. R. Maunsell, and P. Lennie (1990) Coding of image contrast in central visual pathways of macaque. Vision Res. 30 (in press).

Smith, V. C., and J. Pokorny (1975) Spectral sensitivity of the foveal cone photopigments between 400 and $500 \mathrm{~nm}$. Vision Res. 15: 161171.

Spitzer, H., and S. Hochstein (1985a) Simple- and complex-cell response dependences on stimulation parameters. J. Neurophysiol. 53: 1244-1265.

Spitzer, H., and S. Hochstein (1985b) A complex-cell receptive field model. J. Neurophysiol. 53: 1266-1286.

Thorell, L. G., R. L. DeValois, and D. G. Albrecht (1984) Spatial mapping of monkey V1 cells with pure color and luminance stimuli. Vision Res. 24: 751-769. 
Tootell, R. B. H., M. S. Silverman, S. L. Hamilton, R. L. DeValois, and E. Switkes (1988) Functional anatomy of macaque striate cortex. III. Color. J. Neurosci. 8: 1569-1593.

Ts'o, D. Y., and C. D. Gilbert (1988) The organization of chromatic and spatial interactions in the primate striate cortex. J. Neurosci. 8: 1712-1727.

Van der Horst, G. J. C., and M. A. Bouman (1969) Spatiotemporal chromaticity discrimination. J. Opt. Soc. $\Lambda \mathrm{m}$. 59: 1482-1488.

Vautin, R. G., and B. M. Dow (1985) Color cell groups in foveal striate cortex of the behaving macaque. J. Neurophysiol. 54: 273-292.
Vimal, R. L. P., J. Pokorny, V. C. Smith, and S. K. Shevell (1989) Foveal cone thresholds. Vision Res. 29: 61-78.

Walraven, P. L. (1974) A closer look at the tritanopic convergence point. Vision Res. 14: 1339-1343.

Wiesel, T. N., and D. H. Hubel (1966) Spatial and chromatic interactions in the lateral geniculate body of the rhesus monkey. J. Neurophysiol. 29: 1115-1156. 